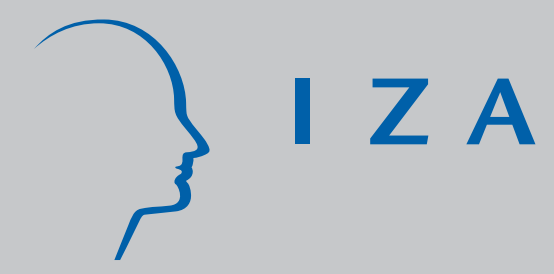

IZA DP No. 3226

Are Immigrants More Mobile Than Natives?

Evidence from Germany

Matthias Schündeln

December 2007 


\title{
Are Immigrants More Mobile Than Natives? Evidence from Germany
}

\author{
Matthias Schündeln \\ Harvard University \\ and IZA
}

Discussion Paper No. 3226

December 2007

\author{
IZA \\ P.O. Box 7240 \\ 53072 Bonn \\ Germany \\ Phone: +49-228-3894-0 \\ Fax: +49-228-3894-180 \\ E-mail: iza@iza.org
}

\begin{abstract}
Any opinions expressed here are those of the author(s) and not those of the institute. Research disseminated by IZA may include views on policy, but the institute itself takes no institutional policy positions.

The Institute for the Study of Labor (IZA) in Bonn is a local and virtual international research center and a place of communication between science, politics and business. IZA is an independent nonprofit company supported by Deutsche Post World Net. The center is associated with the University of Bonn and offers a stimulating research environment through its research networks, research support, and visitors and doctoral programs. IZA engages in (i) original and internationally competitive research in all fields of labor economics, (ii) development of policy concepts, and (iii) dissemination of research results and concepts to the interested public.
\end{abstract}

IZA Discussion Papers often represent preliminary work and are circulated to encourage discussion. Citation of such a paper should account for its provisional character. A revised version may be available directly from the author. 


\section{ABSTRACT \\ Are Immigrants More Mobile Than Natives? Evidence from Germany*}

Low rates of internal migration in many European countries contribute to the persistence of significant regional labor market differences. To further our understanding of the underlying reasons I study internal migration in Germany, using the Mikrozensus, a very large sample of households living in Germany. The first contribution of this paper is to quantify the low mobility of the German population by estimating the unobserved cost of migration. I then focus on the differences between immigrants and natives, and start by presenting reducedform econometric evidence for the hypothesis that immigrants, once they are in the country of destination, are more mobile than natives. Observable, individual-level characteristics can only explain part of this finding. To estimate differences in the responsiveness to labor market characteristics that are due to unobserved characteristics, I then estimate conditional logit models of the migration decision across the German federal states. I find significantly higher responsiveness to labor market differentials in the immigrant population than in the native population. Unobserved moving costs for immigrants are estimated to be only about $37 \%$ of this same cost for natives. The findings bear on the assessment of the economic impact of immigration, and the paper contributes to the current immigration-related policy debates that feature prominently in many European countries, and that likely will continue to be important in light of the ongoing EU expansion and the expected resulting east-west migration.

JEL Classification: J61, R23

Keywords: internal migration, immigrants, cost of migration, regional convergence, Germany

Corresponding author:

Matthias Schündeln

Department of Economics

Harvard University

Littauer Center 1875

Cambridge Street

Cambridge, MA 02138

USA

E-mail: schuendeln@harvard.edu

I would like to thank Joseph Altonji, Nicola Fuchs-Schündeln, Jenny Hunt, Paul Schultz, and Christopher Timmins for helpful comments, the Institute for the Study of Labor (IZA), Bonn, for hospitality, and the Statistische Bundesamt for giving me access to the data. 


\section{Introduction}

This paper studies the interaction between two issues with significant importance for policy: Regional labor market disparities and the role of immigration in the labor market. First, when there are sizeable differences between regions with respect to labor market characteristics, as is the case in many European countries, internal migration might be one way to achieve efficiency-enhancing convergence. ${ }^{1}$ However, rates of internal migration are often low. For example, a recent study by the German Institute for Employment Research (Institut für Arbeitsmarkt- und Berufsforschung, 2002) finds that in a sample of unemployed individuals, $63 \%$ would "by no means" be willing to change their place of residence for a job. ${ }^{2}$ Similarly low willingness to migrate has been documented for Italy by Faini et al. (1997). They find that almost $40 \%$ of the unemployed would not take a job outside the town in which they currently reside. Evidence for low mobility, especially of low skilled individuals, also exists for the UK (Gregg et al. 2004). Thus, labor migration and consequently the convergence due to labor migration will likely be slow in these countries. The first contribution of this paper is to quantify the low mobility of the population by assigning a monetary value to the unobserved cost of migration. I also investigate differences between age groups in their responsiveness to labor market differentials, which have been found to be important in explaining observed behavior at the aggregate level (Hunt 2006). Because of a very large and detailed data set, I can significantly add to existing analyses of this kind.

Second, immigration related-questions feature prominently in current policy debates in many European countries, and likely will continue to be important in light of the ongoing EU expansion towards Eastern Europe and the expected resulting east-west migration. This paper focuses on the within-country migration of immigrants after their initial international migration. It has been hypothesized that immigrants are "likely [...] the most mobile of workers" (Friedberg and Hunt 1995, p. 31) which would imply that a large immigrant population can contribute to an acceleration of convergence in response to regional economic shocks. Immigrants constitute a self-selected group of individuals from the country of origin (Borjas 1987, Chiswick 2000) and may differ systematically, for example with respect to risk aversion, not only from individuals in their native country, but also from individuals in their host country. Immigrants have chosen to incur presumably large costs to move from their native country to the host country, and therefore it may be that they are the most mobile individuals with

\footnotetext{
${ }^{1}$ See Borjas (2001) for a simple model that illustrates the gains from internal migration.

${ }^{2}$ Of the remaining $37 \%, 11 \%$ would be willing to change the place of residence, while $26 \%$ would grudgingly change the place of residence. On the other hand, only $26 \%$ would by no means accept a lower income to find a new job, while $55 \%$ would accept a lower income grudgingly (Institut für Arbeitsmarkt- und Berufsforschung, 2002).
} 
regards to internal migration as well. However, little is known about the differences between immigrants and natives with respect to their internal migration behavior. The few studies that do empirically analyze this question do come to inconclusive results. Notably, a study by Bartel and Koch (1991) examines internal migration decisions of US immigrants. Without quantifying the difference, they find that immigrants' internal mobility rate is comparable to and in some groups higher than that of natives. However, they do not find significant effects of regional economic characteristics on the probability of internal migration. ${ }^{3}$ This paper seeks to fill the existing gap in this literature.

I use a large sample with individual-level data on migration decisions and personal characteristics to test the proposition that immigrants are more mobile than natives. I am also able to quantify the differences in internal mobility between these two groups. The definition of immigrants is not straightforward (see for example the discussion in Pischke and Velling, 1997) and it is useful to clarify the terminology early on. ${ }^{4}$ In this paper, "immigrant" refers to individuals who are foreign born and non-nationals, as opposed to "foreigners", which refers to all resident non-nationals, i.e. both immigrants and individuals born in Germany but not German citizens. On the other hand, "natives" are defined as German citizens who are also born in Germany.

The analysis proceeds in several steps. I first address the main question of the paper: Are immigrants more mobile than natives? Based on a large sample of individual observations, I estimate reduced form models of the determinants of internal migration and test for differences between immigrants and natives. I find strong evidence in favor of the hypothesis that immigrants are more mobile than natives. Because I can control for a large number of observable individual characteristics I can further conclude that these cannot fully explain differences in migration behavior between these groups. Thus, I can rule out that selection based on observable characteristics is underlying the observed differences in the aggregate. In a second step, I then provide some suggestive evidence for other, typically unobserved reasons why migration behavior may differ between these two groups. In the last step of the analysis I investigate the differences in the reaction to labor market conditions and quantify the difference in migration behavior of the two groups under study based on a simple structural model of

\footnotetext{
${ }^{3}$ Kritz and Nogle (1994) compare the probability of internal migration between natives and different immigrant groups. However, they do not explore the differences in the determinants of internal migration decisions that may explain differences in migration probabilities.

${ }^{4}$ Official statistics from the Statistische Bundesamt (the German Federal Statistical Office) follow a definition based on nationality, irrespective of the place of birth. This definition is shared by most other European countries, while in the United States immigrants are defined as foreign born, independent of nationality. Angrist and Kugler (2001) explore possible differences in these definitions and conclude that for most European countries the groups of non-nationals and recently arrived foreign born residents are "roughly coincident" (Angrist and Kugler 2001, p.14).
} 
migration decisions. To this end, I first calculate predicted incomes as well as unemployment probabilities that each individual would face in each of the 16 German Bundesländer (the German federal states). ${ }^{5}$ I then use these predicted values and other state characteristics to estimate conditional logit models of the individual's migration decision between these German federal states.

The results in this paper demonstrate that immigrants are more likely to move within Germany than natives. The reduced form results hold after controlling for individual characteristics such as age, marital status, educational background, and employment status. I further provide some direct survey evidence that, on average, foreigners are less connected to the area that they live in and that, by their own assessment, they are more willing to move. While the direct survey evidence does not allow me to disentangle job and family related reasons, I can provide evidence for the importance of economic conditions in the econometric analysis that builds on an explicit structural model of migration decisions. The results of this analysis concur with the reduced form analysis and the direct survey evidence presented in the first part of the paper. They show much higher responsiveness to labor market differentials by the immigrant population than by the native population. The results are statistically and economically significant. Further, I estimate the unobserved moving costs for immigrants to be only $37 \%$ of the corresponding costs for natives. Combined with the fact that immigrants respond more to labor market differentials, this strongly suggests that they contribute to an acceleration of convergence between regions.

Germany is an important case study because of its large regional disparities and its large size of the immigrant population. Yet the findings are also of interest to other countries with large immigrant populations, such as the United States, in which internal migration is a major determinant of regional demographic changes (e.g. Borjas et al. 1992, Newbold 1999) and a contributor to regional growth, and especially for other European countries in light of the ongoing EU expansion towards Eastern Europe and the expected resulting east-west migration (e.g. Bauer and Zimmermann, 1999).

Studies of the economic impact of immigration typically focus on the effect of immigration on the host country's labor market, in particular on wages and employment of natives. ${ }^{6}$

\footnotetext{
${ }^{5}$ While in the reduced-form part of the analysis I can consider any change of residence, i.e. even the geographically smallest migration behavior, the conditional logit analysis is carried out at the state level (but still allowing for within-state migration). This is done, firstly, because of data requirements (for more on this see the data section). Secondly, one might expect that it is more likely that migration is necessary in response to shocks to larger regions, while the rational reaction in response to shocks to smaller spatial units (e.g. as defined by the Insitut für Arbeitsmarkt- und Berufsforschung) may be commuting rather than migrating.

${ }^{6}$ For an overview of the field of the economics of immigration see for example Friedberg and Hunt (1995), LaLonde and Topel (1997), and Borjas (1999b). In light of ageing populations in many receiving countries there has recently been an increased interest in studying the impact of immigration on social security systems
} 
However, regional labor markets in most countries show considerable diversity. Convergence between markets would typically result in efficiency gains, and it is therefore important to ask what determines convergence, who contributes to convergence, and how the rate of convergence can be increased. In a set of two papers, Borjas (1999a, 2001) investigates the effect of regional differences in welfare and wage levels on the initial migration of newly arriving immigrants to the United States and studies the resulting efficiency gains. Bartel (1989) estimates the determinants of location choice of new immigrants to the United States. Several papers (e.g. Funkhouser 2000, Kritz and Nogle 1994, Newbold 1999) study the secondary migration patterns of immigrants within the United States. The purpose of these papers is to understand whether existing regional concentrations of immigrants are accentuated through internal migration of immigrants, for example due to network effects, which could potentially explain internal migration even in the absence of wage differentials. Regarding the role of labor flows as a means to achieve convergence of economic conditions across regions, Blanchard and Katz (1992) demonstrate the importance of labor migration within the United States. Treyz et al. (1993) find that in the United States the employment probability has a larger impact on net migration than wage rates. On the other hand, Decressin and Fatás (1995) find that in Europe migration is a less important mechanism in the adjustment process to a shock, and it is rather the participation rate that adjusts in reaction to a shock. ${ }^{7}$ Methodologically, the paper that is closest to the present paper is a study by Davies et al. (2001) who estimate conditional logit models of migration between US states, based on aggregate data.

In a setting of high unemployment rates, especially in policy debates, immigration is often seen as placing an additional burden on host countries' labor markets. ${ }^{8}$ Any discussion of economic gains and costs of immigration will have to take into account differences between immigrants and natives in labor market behavior, as for example mobility. If in fact immigrants are more mobile than natives (with respect to internal migration) in response to changing economic conditions, which is what I find, this constitutes a positive economic impact of the

(e.g. von Hagen and Walz 1994). There is also a large body of work on the reasons of emigration and the effect of migration on the country of origin, for example Appleyard (1998). Finally, there is strand of the literature that studies return migration (e.g. Dustmann 2003).

${ }^{7}$ Decressin (1994) studies determinants of migration between West German states before German reunification. He concludes that the unemployment and income variables do not significantly predict migration flows. Puhani (2001) finds that within and cross border labor mobility is unlikely to accommodate unemployment shocks between West Germany, France and Italy. Hunt (2006) focuses on differences in the responsiveness to labor market conditions depending on the age of the individual. She finds that the young are very sensitive to wages in the region of origin, while they are relatively insensitive to unemployment rates in the origin region

${ }^{8}$ The empirical evidence is mixed: for the United States only small employment effects of immigrant arrivals have been found, with some evidence for a wage effect (see Friedberg and Hunt, 1995). For Germany Pischke and Velling (1997) cannot find evidence for a displacement effect of immigrants, while De New and Zimmermann (1994) find an effect on wages. 
immigrant population for the host country, which should be taken into account.

The remainder of the paper is structured as follows: In the following section I describe the sources of the data that are used in this paper, and I give an overview of labor market characteristics. Section 3 presents reduced form evidence that demonstrates that immigrants are more likely to migrate than natives within Germany. The next section provides an intuitive discussion of potential reasons for why immigrants might be more mobile than natives with respect to internal migration. This section also provides some direct survey evidence in support of some hypotheses that are mentioned in the literature. In section 5, I present the model that is used to test between various hypotheses, and that will allow me to quantify the difference in migration responsiveness between immigrants and natives. Then the results of the estimation of this model are presented and discussed. The last section concludes.

\section{The data}

I draw data from three main data sources. First, and foremost, I use individual level data from the German Mikrozensus ("microcensus" from now on). This survey is mandated by German law and run by the German Federal Statistical Office. It is a repeated cross-section that is run every year for a $1 \%$ subsample of the population living in Germany, thus providing me with a very large sample size of about 800,000 observations for each year (before sample selection criteria are applied). Through a special arrangement with the German Federal Statistical Office I am also able to use additional, more restricted, information from the microcensus on internal migration that is available for a $0.45 \%$ subsample of the population. For the estimation of the conditional logit models below, I use the full matrix of migration flows (i.e. inflows as well as outflows) between the 16 German states as implied by the $0.45 \%$ subsample. $^{9}$ I pool the microcensus data for the years 1996-2003.

Second, for some of the initial analysis I also show some descriptive statistics about migration provided by the German Federal Statistical Office. Since people living in Germany are by law required to report any change of address to the smallest local administrative unit (called "Gemeinde") one can calculate the number of individuals who moved between these units based on these official records that cover the total population, without having to rely on a sample. Further, information is available on whether the individual in question is a native or a foreigner. As mentioned before, the data from the German Federal Statistical Office distinguish between resident non-nationals and German citizens. Last, I also use data from

\footnotetext{
${ }^{9}$ The $1 \%$ subsample does not allow the researcher to identify the federal state of origin, only whether an individual moved across borders within the last year. For confidentiality reasons the variable regarding state of origin is only available to researchers under special arrangements.
} 
the German Socio-Economic Panel (GSOEP), an annual household panel survey, for a subset of the analysis.

It is worth commenting on the question why several different sources of data are necessary for this analysis. Although the GSOEP is a panel and provides some more detail on individuals, the microcensus is used to answer the main question of the paper because it is the data source that combines a large sample size with detailed information about the individuals. In the main econometric analysis below I model the decision making process of an individual as a choice between several available federal states with different characteristics and the econometric techniques employed require information about flows between all possible choices. However, because migration is relatively infrequent (see below for more specific evidence), despite the relatively large sample size in GSOEP, less than 900 immigrants migrate across federal state borders in that survey in the sample of 18-60 year old individuals that is used in the analysis below. ${ }^{10}$ With a total of 256 cells (from all 16x16 possible migration flows between the 16 federal states) this results in "empty cells". However, in the underlying structural model empty cells have a probability of zero, and therefore it would not be possible to estimate the conditional logit models, which are estimated with maximum likelihood. On the other hand, the very large sample size of the microcensus actually allows me to further split the sample and analyze, e.g., different age groups separately. Thus, the microcensus is used for the conditional logit analysis. ${ }^{11}$

\section{Background}

The German labor market exhibits large regional disparities with respect to key characteristics, as for example unemployment rates and hourly wages. This is especially true if one compares east versus west German states. However, also within eastern and western states there are large differences. For example, the average unemployment rate in 1998 in the western states (including West Berlin) was 10.5\%, while it was 19.5\% in the eastern states. But unemployment was as low as $8.0 \%$ in Baden-Württemberg, the western state with the lowest unemployment rate, and was more than twice that percentage, namely $16.6 \%$, in Bremen, the western state with the highest unemployment rate. Table 14 in the appendix gives an overview of the relevant labor market characteristics by federal state for a representative year.

Over the decade from 1991 to 2001 the resident non-national population in Germany has increased by almost a quarter: from 5.88 million to 7.32 million (Statistisches Bundesamt 2002). In 1999, the approximate midpoint of the time period used in this study, 7.34 million

\footnotetext{
${ }^{10}$ See also the discussion in Hunt (2006) who uses GSOEP data for her individual-level analysis.

${ }^{11}$ Similar reasons rule out the analysis at a lower level of disaggregation, since one would always require that migration flows are observed for all possible cells, i.e. between all possible origins and destinations.
} 
people, that is $9 \%$ of the total German population, belonged to this group. Among them the largest group, namely 2.11 million, came from Turkey (see table 15 in the appendix). The second and third largest groups were coming from Yugoslavia and Italy, with 0.719 and 0.612 million people respectively. Table 16 in the appendix shows how the foreigners are distributed among the German federal states. Again, we note large disparities between the states, with the largest absolute numbers of foreigners in Nordrhein-Westfalen $(1,995,000)$ and Baden-Württemberg $(1,267,000)$. In relative numbers, Bremen and Hamburg have the highest proportion of foreigners, with foreigners constituting $15.2 \%$ of the total population in these two federal states. On the other extreme, Mecklenburg-Vorpommern's foreign population constitutes only $1.7 \%$ of the total population in 1999 , with only 26,000 foreigners.

\section{Reduced form evidence using microcensus data}

A simple look at descriptive evidence using official registration records demonstrates that foreigners living in Germany are more mobile than Germans citizens. In the appendix (see appendix B) I present data that shows that differences between natives and foreigners exist with respect to migration across all regional levels, namely across Gemeinde- (town) borders, Kreis- (county) borders and Länder- (federal state) borders (there are 16 Länder, 439 Kreise and several thousand Gemeinden). The problem with this first rough analysis is obviously that it does not control for any individual characteristics, and it is likely that observable differences, for example, in the age distribution or in the level of education, can explain part of these differences. A second issue is that the official records do not allow me to distinguish between foreigners born in Germany and those that immigrated from abroad.

Therefore, I now turn to the question whether immigrants are more likely to migrate holding a large number of potentially migration-relevant individual characteristics constant, which the microcensus allows me to do. Using the microcensus data I am also able to identify "immigrants" as opposed to a broader definition of "foreigners" (the latter includes individuals who were born in Germany, but do not have German citizenship).

For this analysis, I therefore keep only individuals who are German citizens and born in Germany, the subsample which I will call "natives", as well as non-nationals (those without German citizenship) that were not born in Germany, the subsample that I will refer to as "immigrants". Thus, I exclude non-nationals that were born in Germany and German nationals that were born outside of Germany. I also drop individuals that have dual citizenship (i.e. German and another citizenship). Consequently, the analysis in this section is based only on the groups immigrants and natives in their most narrow definition. 
I further restrict the sample as follows: (1) I use the sample of individuals of age 18-60. Dropping older individuals deals with issues of self-selection into early retirement that one might encounter if individuals up to age 65 are included. Note that I also do some of the analysis separate by age group. (2) I also drop farm households. In addition to the obvious expectation that they are very immobile, this is done because income data is not available for farm households. (3) Individuals who migrated to Germany from abroad during the last year are dropped from the analysis. (4) I drop individuals who are currently in school. My goal is to study the role of labor market characteristics, and migration related to education would confound the results. ${ }^{12}$ Below, I also perform some robustness checks with a sample that focuses on household heads that are labor market participants (i.e. including unemployed) and not working part time.

The microcensus provides information on (a) whether an individual changed her "place of residence" as well as (b) whether the individual migrated from another German federal state. I start with the analysis of the broader definition of migration. The dependent variable is thus a dummy variable which is one if an individual changed her "place of residence" within the last 12 months. Therefore, this part of the analysis does take into account all possible moves, not only migration across federal state borders.

Because of the large number of observations, I can control very flexibly for household characteristics and separately identify a large number of indicator variables. Therefore, I create indicator variables for schooling and higher educational as well as vocational training levels (four indicator variables, omitted category is "not finished school") ${ }^{13}$, three indicators for marital status (omitted category is "single"), and indicator variables for each of ten household size groups. I further create indicator variables for 10 different income classes, split at the deciles of the income distribution. Income is measured at the individual level from the microcensus as follows: For each individual the data provide her (net) income bracket, with fairly narrow brackets for the relevant income range. I take the mid-point of a bracket as the income estimate. Incomes are deflated to year 2000 DM values. ${ }^{14}$ I pool data for 1996 to 2003, and also include year dummies.

The following tables show results from probit regressions (shown are the marginal coefficients). Table 1 shows in column 1 that immigrants are 9 percentage points more likely than

\footnotetext{
${ }^{12}$ The results are substantially unchanged if individuals that are currently in school are included in the analysis.

${ }^{13}$ The indicators are aggregating different schooling levels as follows: "finished school" indicates that the individual has any degree from a school. "vocational training" implies a lower level of vocational training (the German levels "Praktikum" or "Lehre" or "Berufsfachschule") while "higher vocational training" comprises the German levels "Meister" and "Fachschule". "Tertiary degree" includes all college-level degrees.

${ }^{14}$ Values stated in Euros (in 2003) are expressed in DM terms and deflated.
} 
natives to change their place of residence over a 1 year time horizon. Adding controls to this baseline result, namely age and age squared (age is rescaled by dividing by 100), marital status and variables related to their level of education, reduces the difference between immigrants and natives somewhat, namely to 7.6 percentage points (column 4). This indicates that immigrants are more likely to have observable characteristics, such as being of relatively young age, that are associated with higher probability of migrating. However, these characteristics can only explain about 1.5 percentage points of the difference between immigrants and natives observed in the baseline. Adding household size indicators (10 indicators) and income categories (10 groups) further reduces the difference between immigrants and natives. Although the survey is not a panel, there is some limited amount of information about the individual's situation one year ago. One is the migration information that I exploit. Another is whether the individual was unemployed a year ago. The indicator variable for unemployment last period has the expected sign, i.e. individuals who were unemployed a year ago are more likely to have moved. However, even after controlling for a large number of characteristics, the difference is still 5.8 percentage points in the most comprehensive specification. 


\begin{tabular}{|c|c|c|c|c|c|c|c|c|}
\hline & (1) & $(2)$ & (3) & (4) & (5) & $(6)$ & (7) & $(8)$ \\
\hline immigrant & $\begin{array}{l}0.090 \\
(0.001)^{* *}\end{array}$ & $\begin{array}{l}0.072 \\
(0.001)^{* *}\end{array}$ & $\begin{array}{l}0.079 \\
(0.001)^{* *}\end{array}$ & $\begin{array}{l}0.076 \\
(0.001)^{* *}\end{array}$ & $\begin{array}{l}0.072 \\
(0.001)^{* *}\end{array}$ & $\begin{array}{l}0.070 \\
(0.002) * *\end{array}$ & $\begin{array}{l}0.058 \\
(0.002) * *\end{array}$ & $\begin{array}{l}0.058 \\
(0.002) * *\end{array}$ \\
\hline age (/100) & & $\begin{array}{l}-0.637 \\
(0.015)^{* *}\end{array}$ & $\begin{array}{l}-0.540 \\
(0.016)^{* *}\end{array}$ & $\begin{array}{l}-0.609 \\
(0.016)^{* *}\end{array}$ & $\begin{array}{l}-0.516 \\
(0.016) * *\end{array}$ & $\begin{array}{l}-0.531 \\
(0.018) * *\end{array}$ & $\begin{array}{l}-0.500 \\
(0.020)^{* *}\end{array}$ & $\begin{array}{l}-0.500 \\
(0.020)^{* *}\end{array}$ \\
\hline age squared & & $\begin{array}{l}0.260 \\
(0.019)^{* *}\end{array}$ & $\begin{array}{l}0.160 \\
(0.019) * *\end{array}$ & $\begin{array}{l}0.233 \\
(0.020)^{* *}\end{array}$ & $\begin{array}{l}0.034 \\
(0.020)\end{array}$ & $\begin{array}{l}0.040 \\
(0.022)\end{array}$ & $\begin{array}{l}0.018 \\
(0.024)\end{array}$ & $\begin{array}{l}0.019 \\
(0.024)\end{array}$ \\
\hline married & & & $\begin{array}{l}-0.018 \\
(0.001)^{* *}\end{array}$ & $\begin{array}{l}-0.016 \\
(0.001)^{* *}\end{array}$ & $\begin{array}{l}0.017 \\
(0.001)^{* *}\end{array}$ & $\begin{array}{l}0.017 \\
(0.001)^{* *}\end{array}$ & $\begin{array}{l}0.016 \\
(0.001)^{* *}\end{array}$ & $\begin{array}{l}0.015 \\
(0.001)^{* *}\end{array}$ \\
\hline widowed & & & $\begin{array}{l}0.015 \\
(0.002)^{* *}\end{array}$ & $\begin{array}{l}0.019 \\
(0.002)^{* *}\end{array}$ & $\begin{array}{l}0.029 \\
(0.002)^{* *}\end{array}$ & $\begin{array}{l}0.027 \\
(0.002) * *\end{array}$ & $\begin{array}{l}0.026 \\
(0.003)^{* *}\end{array}$ & $\begin{array}{l}0.025 \\
(0.003) * *\end{array}$ \\
\hline divorced & & & $\begin{array}{l}0.046 \\
(0.001)^{* *}\end{array}$ & $\begin{array}{l}0.050 \\
(0.001)^{* *}\end{array}$ & $\begin{array}{l}0.055 \\
(0.001)^{* *}\end{array}$ & $\begin{array}{l}0.054 \\
(0.001)^{* *}\end{array}$ & $\begin{array}{l}0.051 \\
(0.001)^{* *}\end{array}$ & $\begin{array}{l}0.051 \\
(0.001)^{* *}\end{array}$ \\
\hline finished school & & & & $\begin{array}{l}0.001 \\
(0.002)\end{array}$ & $\begin{array}{l}-0.005 \\
(0.002)^{* *}\end{array}$ & $\begin{array}{l}-0.005 \\
(0.002)^{* *}\end{array}$ & $\begin{array}{l}-0.005 \\
(0.002)^{*}\end{array}$ & $\begin{array}{l}-0.004 \\
(0.002)^{*}\end{array}$ \\
\hline vocational training & & & & $\begin{array}{l}-0.003 \\
(0.002)\end{array}$ & $\begin{array}{l}-0.011 \\
(0.002)^{* *}\end{array}$ & $\begin{array}{l}-0.012 \\
(0.002)^{* *}\end{array}$ & $\begin{array}{l}-0.008 \\
(0.002)^{* *}\end{array}$ & $\begin{array}{l}-0.009 \\
(0.002) * *\end{array}$ \\
\hline higher voc. training & & & & $\begin{array}{l}0.001 \\
(0.002)\end{array}$ & $\begin{array}{l}-0.008 \\
(0.002)^{* *}\end{array}$ & $\begin{array}{l}-0.007 \\
(0.002)^{* *}\end{array}$ & $\begin{array}{l}-0.005 \\
(0.002)^{*}\end{array}$ & $\begin{array}{l}-0.005 \\
(0.002)^{*}\end{array}$ \\
\hline tertiary degree & & & & $\begin{array}{l}0.023 \\
(0.002)^{* *}\end{array}$ & $\begin{array}{l}0.010 \\
(0.002)^{* *}\end{array}$ & $\begin{array}{l}0.012 \\
(0.002)^{* *}\end{array}$ & $\begin{array}{l}0.010 \\
(0.002)^{* *}\end{array}$ & $\begin{array}{l}0.010 \\
(0.002)^{* *}\end{array}$ \\
\hline unemployed last yr & & & & & & & $\begin{array}{l}0.030 \\
(0.001)^{* *}\end{array}$ & $\begin{array}{l}0.030 \\
(0.001) * *\end{array}$ \\
\hline $\begin{array}{l}\text { household size } \\
\text { indicators (10) }\end{array}$ & & & & & yes & yes & yes & yes \\
\hline $\begin{array}{l}\text { income category } \\
\text { indicators (10) }\end{array}$ & & & & & & yes & yes & yes \\
\hline year indicators (8) & & & & & & & & yes \\
\hline observations & 1266076 & 1266076 & 1266076 & 1229115 & 1225052 & 1076277 & 942942 & 923942 \\
\hline log-likelihood & -360689.4 & -338358.8 & -335805.7 & -326195.6 & -317354.9 & -285563.0 & -244707.9 & -244227.2 \\
\hline
\end{tabular}

Table 1: Reduced form results from the microcensus 
Many of the immigrants in Germany have been in the country for many years. For policy purposes, however, we might be interested in the effect of current and future immigration. To approximate the labor market behavior of these immigrant flows, I study recent immigrants, defined as those who have been living in Germany for less then 10 years at the time of the microcensus. The baseline results show a much larger propensity of recent immigrants to migrate than both immigrants as a whole and natives have. Recent immigrants are 20 percentage points more likely to have changed their place of residence than are natives. This implies that they are about 11 percentage points more likely than all immigrants to change their place of residence within a year. However, once all observable characteristics are taken into account, this effect is approximately cut by half. Simply taking age into account already drops the coefficient from 0.202 to 0.129 , thus the fact that recent immigrants are younger on average explains a large portion of the observed difference. Nevertheless, even after controlling for a large set of characteristics, recent immigrants are still about 9 percentage points more likely to migrate than natives, more than 50\% higher than the difference that is observed between all immigrants and natives.

\begin{tabular}{|c|c|c|c|c|c|c|c|c|}
\hline \multicolumn{9}{|c|}{ Dependent variable $=1$ if individual changed place of residence over the previous year } \\
\hline & $(1)$ & $(2)$ & $(3)$ & $(4)$ & (5) & (6) & (7) & $(8)$ \\
\hline recent immigrant & $\begin{array}{l}0.202 \\
(0.003)^{* *}\end{array}$ & $\begin{array}{l}0.129 \\
(0.002)^{* *}\end{array}$ & $\begin{array}{l}0.140 \\
(0.002)^{* *}\end{array}$ & $\begin{array}{l}0.129 \\
(0.002) * *\end{array}$ & $\begin{array}{l}0.112 \\
(0.002)^{* *}\end{array}$ & $\begin{array}{l}0.112 \\
(0.003)^{* *}\end{array}$ & $\begin{array}{l}0.091 \\
(0.003) * *\end{array}$ & $\begin{array}{l}0.091 \\
(0.003) * *\end{array}$ \\
\hline age $(/ 100)$ & & $\begin{array}{l}-0.607 \\
(0.015)^{* *}\end{array}$ & $\begin{array}{l}-0.508 \\
(0.016)^{* *}\end{array}$ & $\begin{array}{l}-0.566 \\
(0.016)^{* *}\end{array}$ & $\begin{array}{l}-0.481 \\
(0.016)^{* *}\end{array}$ & $\begin{array}{l}-0.514 \\
(0.018)^{* *}\end{array}$ & $\begin{array}{l}-0.491 \\
(0.020)^{* *}\end{array}$ & $\begin{array}{l}-0.491 \\
(0.020)^{* *}\end{array}$ \\
\hline age squared & & $\begin{array}{l}0.237 \\
(0.019) * *\end{array}$ & $\begin{array}{l}0.136 \\
(0.019)^{* *}\end{array}$ & $\begin{array}{l}0.194 \\
(0.020) * *\end{array}$ & $\begin{array}{l}0.004 \\
(0.020)\end{array}$ & $\begin{array}{l}0.030 \\
(0.022)\end{array}$ & $\begin{array}{l}0.014 \\
(0.024)\end{array}$ & $\begin{array}{l}0.015 \\
(0.024)\end{array}$ \\
\hline married & & & $\begin{array}{l}-0.018 \\
(0.001)^{* *}\end{array}$ & $\begin{array}{l}-0.016 \\
(0.001)^{* *}\end{array}$ & $\begin{array}{l}0.016 \\
(0.001)^{* *}\end{array}$ & $\begin{array}{l}0.017 \\
(0.001)^{* *}\end{array}$ & $\begin{array}{l}0.016 \\
(0.001)^{* *}\end{array}$ & $\begin{array}{l}0.016 \\
(0.001)^{* *}\end{array}$ \\
\hline widowed & & & $\begin{array}{l}0.014 \\
(0.002)^{* *}\end{array}$ & $\begin{array}{l}0.017 \\
(0.002)^{* *}\end{array}$ & $\begin{array}{l}0.028 \\
(0.002)^{* *}\end{array}$ & $\begin{array}{l}0.026 \\
(0.002)^{* *}\end{array}$ & $\begin{array}{l}0.025 \\
(0.003) * *\end{array}$ & $\begin{array}{l}0.025 \\
(0.003) * *\end{array}$ \\
\hline divorced & & & 0.045 & $\begin{array}{l}0.049 \\
(0.001)^{* *}\end{array}$ & $\begin{array}{l}0.055 \\
(0.001)^{* *}\end{array}$ & $\begin{array}{l}0.054 \\
(0.001)^{* *}\end{array}$ & $\begin{array}{l}0.051 \\
(0.001)^{* *}\end{array}$ & $\begin{array}{l}0.051 \\
(0.001)^{* *}\end{array}$ \\
\hline finished school & & & & $\begin{array}{l}-0.004 \\
(0.002)^{* *}\end{array}$ & $\begin{array}{l}-0.011 \\
(0.001)^{* *}\end{array}$ & $\begin{array}{l}-0.011 \\
(0.002)^{* *}\end{array}$ & $\begin{array}{l}-0.011 \\
(0.002)^{* *}\end{array}$ & $\begin{array}{l}-0.011 \\
(0.002)^{* *}\end{array}$ \\
\hline vocational training & & & & $\begin{array}{l}-0.011 \\
(0.002)^{* *}\end{array}$ & $\begin{array}{l}-0.020 \\
(0.002)^{* *}\end{array}$ & $\begin{array}{l}-0.021 \\
(0.002)^{* *}\end{array}$ & $\begin{array}{l}-0.018 \\
(0.002)^{* *}\end{array}$ & $\begin{array}{l}-0.018 \\
(0.002)^{* *}\end{array}$ \\
\hline higher voc. training & & & & $\begin{array}{l}-0.008 \\
(0.002)^{* *}\end{array}$ & $\begin{array}{l}-0.016 \\
(0.001)^{* *}\end{array}$ & $\begin{array}{l}-0.016 \\
(0.002)^{* *}\end{array}$ & $\begin{array}{l}-0.014 \\
(0.002)^{* *}\end{array}$ & $\begin{array}{l}-0.014 \\
(0.002)^{* *}\end{array}$ \\
\hline tertiary degree & & & & $\begin{array}{l}0.013 \\
(0.002)^{* *}\end{array}$ & $\begin{array}{l}-0.000 \\
(0.002)\end{array}$ & $\begin{array}{l}0.002 \\
(0.002)\end{array}$ & $\begin{array}{l}-0.000 \\
(0.002)\end{array}$ & $\begin{array}{l}-0.001 \\
(0.002)\end{array}$ \\
\hline unemployed last yr & & & & & & & $\begin{array}{l}0.030 \\
(0.001) * *\end{array}$ & $\begin{array}{l}0.030 \\
(0.001)^{* *}\end{array}$ \\
\hline $\begin{array}{l}\text { household size } \\
\text { indicators (10) }\end{array}$ & & & & & yes & yes & yes & yes \\
\hline $\begin{array}{l}\text { income category } \\
\text { indicators (10) }\end{array}$ & & & & & & yes & yes & yes \\
\hline year indicators $(8)$ & & & & & & & & yes \\
\hline Observations & 1266076 & 1266076 & 1266076 & 1229115 & 1225052 & 1076277 & 942942 & 942942 \\
\hline log-likelihood & -358439.1 & -337649.4 & -335116.4 & -325617.6 & -317115.1 & -285375.9 & -244692.3 & -244211.5 \\
\hline
\end{tabular}

Table 2: Reduced form results from the microcensus: compare natives to recent immigrants 
So far, I have only allowed for a constant difference between immigrants and natives. However, both groups may also differ with respect to the role that other variables play in the decision to migrate. Next, I therefore estimate probit regressions separately for natives, immigrants and recent immigrants (see table 3). In these regressions I include a full set of dummies for marital status, highest educational and vocational training levels, household size and income bracket. While a Chow test rejects that all the coefficients are jointly equal, there are some individual variables for which differences do not seem to be very pronounced. In particular the parameter estimate for the age variable is of similar size for natives and immigrants (it is somewhat more different for recent immigrants). On the other hand, I find larger differences with respect to the education variables. Also, the role of the family situation of the individual differs substantially between the three groups. There are also noteworthy differences with respect to last period's unemployment status: immigrants are more responsive to unemployment shocks than natives, recent immigrants show the largest responsiveness.

\begin{tabular}{|c|c|c|c|c|}
\hline & $\begin{array}{l}\text { (1) } \\
\text { all }\end{array}$ & $\begin{array}{l}(2) \\
\text { natives }\end{array}$ & $\begin{array}{l}\text { (3) } \\
\text { immigrants }\end{array}$ & $\begin{array}{l}\text { (4) } \\
\text { recent immigrants }\end{array}$ \\
\hline \multirow[t]{2}{*}{ age (/100) } & -0.517 & -0.504 & -0.490 & -1.004 \\
\hline & $(0.020) * *$ & $(0.020)^{* *}$ & $(0.128)^{* *}$ & $(0.287)^{* *}$ \\
\hline \multirow[t]{2}{*}{ age squared } & 0.026 & 0.031 & -0.204 & 0.837 \\
\hline & $(0.024)$ & $(0.024)$ & $(0.160)$ & $(0.384)^{*}$ \\
\hline \multirow[t]{2}{*}{ married } & 0.019 & 0.016 & 0.013 & -0.012 \\
\hline & $(0.001) * *$ & $(0.001) * *$ & $(0.005)^{* *}$ & $(0.010)$ \\
\hline \multirow[t]{2}{*}{ widowed } & 0.029 & 0.027 & 0.002 & -0.051 \\
\hline & $(0.003) * *$ & $(0.003) * *$ & $(0.015)$ & $(0.034)$ \\
\hline \multirow[t]{2}{*}{ divorced } & 0.054 & 0.053 & 0.014 & 0.003 \\
\hline & $(0.001)^{* *}$ & $(0.001)^{* *}$ & $(0.008)$ & $(0.017)$ \\
\hline finished school & $\begin{array}{l}-0.017 \\
(0.002)^{* *}\end{array}$ & $\begin{array}{l}-0.008 \\
(0.003) * *\end{array}$ & $\begin{array}{l}-0.006 \\
(0.005)\end{array}$ & $\begin{array}{l}0.021 \\
(0.013)\end{array}$ \\
\hline vocational training & $\begin{array}{l}-0.030 \\
(0.002)^{* *}\end{array}$ & $\begin{array}{l}-0.014 \\
(0.003)^{* *}\end{array}$ & $\begin{array}{l}0.009 \\
(0.006)\end{array}$ & $\begin{array}{l}0.038 \\
(0.013) * *\end{array}$ \\
\hline higher voc. training & $\begin{array}{l}-0.023 \\
(0.002) * *\end{array}$ & $\begin{array}{l}-0.010 \\
(0.003)^{* *}\end{array}$ & $\begin{array}{l}0.022 \\
(0.010)^{*}\end{array}$ & $\begin{array}{l}0.039 \\
(0.021)\end{array}$ \\
\hline tertiary degree & $\begin{array}{l}-0.009 \\
(0.002) * *\end{array}$ & $\begin{array}{l}0.004 \\
(0.003)\end{array}$ & $\begin{array}{l}0.047 \\
(0.008) * *\end{array}$ & $\begin{array}{l}0.051 \\
(0.016) * *\end{array}$ \\
\hline UE last year & $\begin{array}{l}0.032 \\
(0.001)^{* *}\end{array}$ & $\begin{array}{l}0.030 \\
(0.001) * *\end{array}$ & $\begin{array}{l}0.044 \\
(0.001)^{* *}\end{array}$ & $\begin{array}{l}0.056 \\
(0.001)^{* *}\end{array}$ \\
\hline $\begin{array}{l}\text { household size } \\
\text { indicators (10) }\end{array}$ & yes & yes & yes & yes \\
\hline $\begin{array}{r}\text { income category } \\
\text { indicators }(10)\end{array}$ & yes & yes & yes & yes \\
\hline observations & 942942 & 897571 & 45371 & 15957 \\
\hline log-likelihood & -245603.1 & -227029.2 & -17526.3 & -8704.1 \\
\hline
\end{tabular}

Table 3: Reduced form results from the microcensus: natives and immigrants separately 
The second part of this reduced form analysis considers only migration across borders of the federal state (see table 4). Here the dependent variable is a dummy variable which is one if an individual moved within the last 12 months from another German federal state to the current federal state of residence, and zero otherwise. The baseline probability that an individual migrates to a different federal state is smaller, which also translates into smaller differences between immigrants and natives. However, the difference is still statistically highly significant, even after controlling for a large number of covariates. The difference between immigrants and recent immigrants is larger in relative terms than before.

Considering each group separately reveals that the differences are more pronounced in the inter-state migration than in the analysis of all migration, that includes migratory movements over smaller distances. For example, for the inter-state migration, the age effect of immigrants (and recent immigrants) is actually insignificant in the full sample. Similarly, the family situation does not appear to play a role for immigrants, while it is significant for natives: married, widowed or divorced immigrants do not have significantly different propensities to migrate than single immigrants. 


\begin{tabular}{|c|c|c|c|c|c|c|}
\hline \multicolumn{7}{|c|}{ Dependent variable $=1$ if individual moved to a different federal state over the previous year } \\
\hline & $\begin{array}{l}(1) \\
\text { all }\end{array}$ & $\begin{array}{l}(2) \\
\text { all }\end{array}$ & $\begin{array}{l}(3) \\
\text { all }\end{array}$ & $\begin{array}{l}(4) \\
\text { natives }\end{array}$ & $\begin{array}{l}\text { (5) } \\
\text { immigrants }\end{array}$ & $\begin{array}{l}(6) \\
\text { recent } \\
\text { immigrant }\end{array}$ \\
\hline immigrant & $\begin{array}{l}0.003 \\
(0.000) * *\end{array}$ & $\begin{array}{l}0.003 \\
(0.000) * *\end{array}$ & & & & \\
\hline $\begin{array}{l}\text { recent immigrant } \\
\quad(<10 \mathrm{yrs})\end{array}$ & & & $\begin{array}{l}0.005 \\
(0.001)^{* *}\end{array}$ & & & \\
\hline age $(/ 100)$ & $\begin{array}{l}-0.069 \\
(0.005)^{* *}\end{array}$ & $\begin{array}{l}-0.054 \\
(0.006)^{* *}\end{array}$ & $\begin{array}{l}-0.054 \\
(0.006)^{* *}\end{array}$ & $\begin{array}{l}-0.057 \\
(0.006)^{* *}\end{array}$ & $\begin{array}{l}-0.014 \\
(0.034)\end{array}$ & $\begin{array}{l}0.100 \\
(0.105)\end{array}$ \\
\hline age squared & $\begin{array}{l}0.024 \\
(0.007)^{* *}\end{array}$ & $\begin{array}{l}-0.001 \\
(0.008)\end{array}$ & $\begin{array}{l}-0.001 \\
(0.008)\end{array}$ & $\begin{array}{l}0.002 \\
(0.008)\end{array}$ & $\begin{array}{l}-0.063 \\
(0.044)\end{array}$ & $\begin{array}{l}-0.241 \\
(0.144)\end{array}$ \\
\hline married & $\begin{array}{l}-0.003 \\
(0.000)^{* *}\end{array}$ & $\begin{array}{l}0.001 \\
(0.000) * *\end{array}$ & $\begin{array}{l}0.001 \\
(0.000) * *\end{array}$ & $\begin{array}{l}0.001 \\
(0.000)^{* *}\end{array}$ & $\begin{array}{l}0.002 \\
(0.001)\end{array}$ & $\begin{array}{l}0.001 \\
(0.003)\end{array}$ \\
\hline widowed & $\begin{array}{l}0.001 \\
(0.001)\end{array}$ & $\begin{array}{l}0.002 \\
(0.001)^{*}\end{array}$ & $\begin{array}{l}0.002 \\
(0.001)^{*}\end{array}$ & $\begin{array}{l}0.002 \\
(0.001)^{*}\end{array}$ & $\begin{array}{l}-0.002 \\
(0.004)\end{array}$ & $\begin{array}{l}-0.006 \\
(0.011)\end{array}$ \\
\hline divorced & $\begin{array}{l}0.004 \\
(0.000)^{* *}\end{array}$ & $\begin{array}{l}0.004 \\
(0.000)^{* *}\end{array}$ & $\begin{array}{l}0.004 \\
(0.000)^{* *}\end{array}$ & $\begin{array}{l}0.004 \\
(0.000)^{* *}\end{array}$ & $\begin{array}{l}0.001 \\
(0.002)\end{array}$ & $\begin{array}{l}0.001 \\
(0.006)\end{array}$ \\
\hline finished school & $\begin{array}{l}0.002 \\
(0.001)^{* *}\end{array}$ & $\begin{array}{l}0.001 \\
(0.001)\end{array}$ & $\begin{array}{l}0.001 \\
(0.001)\end{array}$ & $\begin{array}{l}0.002 \\
(0.001)\end{array}$ & $\begin{array}{l}0.000 \\
(0.002)\end{array}$ & $\begin{array}{l}0.004 \\
(0.005)\end{array}$ \\
\hline vocational training & $\begin{array}{l}0.002 \\
(0.001)^{* *}\end{array}$ & $\begin{array}{l}0.001 \\
(0.001)\end{array}$ & $\begin{array}{l}0.001 \\
(0.001)\end{array}$ & $\begin{array}{l}0.001 \\
(0.001)\end{array}$ & $\begin{array}{l}0.002 \\
(0.002)\end{array}$ & $\begin{array}{l}0.006 \\
(0.005)\end{array}$ \\
\hline higher voc. training & $\begin{array}{l}0.005 \\
(0.001)^{* *}\end{array}$ & $\begin{array}{l}0.003 \\
(0.001)^{* *}\end{array}$ & $\begin{array}{l}0.002 \\
(0.001)^{*}\end{array}$ & $\begin{array}{l}0.003 \\
(0.001)^{*}\end{array}$ & $\begin{array}{l}0.008 \\
(0.004)^{*}\end{array}$ & $\begin{array}{l}0.023 \\
(0.011)^{*}\end{array}$ \\
\hline tertiary degree & $\begin{array}{l}0.015 \\
(0.001)^{* *}\end{array}$ & $\begin{array}{l}0.009 \\
(0.001)^{* *}\end{array}$ & $\begin{array}{l}0.008 \\
(0.001)^{* *}\end{array}$ & $\begin{array}{l}0.009 \\
(0.002)^{* *}\end{array}$ & $\begin{array}{l}0.012 \\
(0.003)^{* *}\end{array}$ & $\begin{array}{l}0.021 \\
(0.008)^{* *}\end{array}$ \\
\hline unempl. last yr & & $\begin{array}{l}0.005 \\
(0.000) * *\end{array}$ & $\begin{array}{l}0.005 \\
(0.000) * *\end{array}$ & $\begin{array}{l}0.005 \\
(0.000)^{* *}\end{array}$ & $\begin{array}{l}0.006 \\
(0.002)^{* *}\end{array}$ & $\begin{array}{l}0.011 \\
(0.004)^{* *}\end{array}$ \\
\hline $\begin{array}{l}\text { household size } \\
\text { indicators (10) }\end{array}$ & & yes & yes & yes & yes & yes \\
\hline $\begin{array}{r}\text { income category } \\
\text { indicators }(10)\end{array}$ & & yes & yes & yes & yes & yes \\
\hline Observations & 1219667 & 937733 & 937733 & 893518 & 44215 & 14872 \\
\hline log-likelihood & -66134.4 & -48386.2 & -48374.6 & -45573.0 & -2794.6 & -1625.8 \\
\hline
\end{tabular}

Table 4: Reduced form results from the microcensus: migration across federal states 
Finally, I investigate whether the difference between immigrants and natives is restricted to a specific age group (table 5). I do find smaller differences between immigrants and natives for young individuals (age 18-25) and for the oldest age group ( $>45$ years old). However, immigrants are significantly more likely to migrate than natives over the whole age distribution.

\begin{tabular}{|c|c|c|c|c|}
\hline \multicolumn{5}{|c|}{ Dependent variable $=1$ if individual changed place of residence over the previous year } \\
\hline & (1) & (2) & (3) & (4) \\
\hline & $18-24$ years & 25-34 years & $35-44$ years & $>=45$ years \\
\hline \multirow[t]{2}{*}{ immigrant } & 0.063 & 0.078 & 0.068 & 0.039 \\
\hline & $(0.009) * *$ & $(0.004)^{* *}$ & $(0.003)^{* *}$ & $(0.002)^{* *}$ \\
\hline \multirow[t]{2}{*}{ age $(/ 100)$} & 2.685 & -1.893 & 0.208 & -0.591 \\
\hline & $(2.475)$ & $(0.592)^{* *}$ & $(0.500)$ & $(0.193)^{* *}$ \\
\hline \multirow[t]{2}{*}{ age squared } & -9.169 & 1.741 & -0.904 & 0.369 \\
\hline & $(5.704)$ & $(0.998)$ & $(0.635)$ & $(0.183)^{*}$ \\
\hline \multirow[t]{2}{*}{ married } & 0.081 & 0.015 & 0.005 & 0.001 \\
\hline & $(0.006)^{* *}$ & $(0.002)^{* *}$ & $(0.001)^{* *}$ & $(0.001)$ \\
\hline \multirow[t]{2}{*}{ divorced } & 0.102 & 0.069 & 0.049 & 0.020 \\
\hline & $(0.030)^{* *}$ & $(0.004)^{* *}$ & $(0.002)^{* *}$ & $(0.002)^{* *}$ \\
\hline \multirow[t]{2}{*}{ finished school } & 0.007 & -0.001 & -0.005 & -0.004 \\
\hline & $(0.012)$ & $(0.007)$ & $(0.004)$ & $(0.002)$ \\
\hline \multirow[t]{2}{*}{ vocational training } & -0.014 & -0.006 & -0.007 & -0.004 \\
\hline & $(0.012)$ & $(0.007)$ & $(0.004)$ & $(0.002)$ \\
\hline \multirow[t]{2}{*}{ higher voc. training } & -0.007 & -0.003 & -0.007 & -0.001 \\
\hline & $(0.014)$ & $(0.007)$ & $(0.004)$ & $(0.003)$ \\
\hline \multirow[t]{2}{*}{ tertiary degree } & -0.008 & 0.036 & 0.008 & 0.004 \\
\hline & $(0.016)$ & $(0.008)^{* *}$ & $(0.004)$ & $(0.003)$ \\
\hline \multirow[t]{2}{*}{ unempl. last yr } & 0.036 & 0.045 & 0.033 & 0.017 \\
\hline & $(0.006)^{* *}$ & $(0.003)^{* *}$ & $(0.002)^{* *}$ & $(0.001)^{* *}$ \\
\hline $\begin{array}{l}\text { household size } \\
\text { indicators (10) }\end{array}$ & yes & yes & yes & yes \\
\hline $\begin{array}{l}\text { income category } \\
\text { indicators }(10)\end{array}$ & yes & yes & yes & yes \\
\hline observations & 58070 & 232398 & 293389 & 331667 \\
\hline log-likelihood & -24507.1 & -91655.1 & -73566.9 & -48082.9 \\
\hline
\end{tabular}

Table 5: Reduced form results from the microcensus: by age group

I also check the robustness of the results using a more restricted sample. For the "household head/labor market participants" sample I keep only the head of the household (defined as the reference person in the household). For this sample I further drop individuals for which wage income is not their main source of income, and those that are either working part-time or not participating in the labor market at all. The results, which are reported in the appendix (table 18), indicate that the main findings are robust.

Summarizing, the results in this section demonstrate that immigrants are significantly more likely to migrate within Germany than natives, even after controlling for differences in demographics and in socio-economic characteristics of the individuals. Therefore, observable individual characteristics cannot fully explain the differences. The estimates imply that, holding these characteristics constant, the probability of migration is between 5.8 and 9 per- 
centage points higher for immigrants than for natives. The higher migration response to unemployment last period points towards the importance of labor market characteristics in partly explaining the differences between immigrants and natives. This hypothesis will be explored further in the following sections.

\section{Why could there be differences between natives and immigrants with respect to internal migration?}

Why may the probability of migration be larger for immigrants than for natives as demonstrated in the above empirical work, even after controlling for observable individual characteristics? First, immigrants may have lower social and cultural ties that are specific to the region they are living in. Second, immigrants constitute a self-selected group of individuals from the country of origin. They may differ systematically not only from other individuals in their native country but also from other individuals in their host country, in particular these differences may be with respect to inherently unobservable characteristics (such as risk aversion, or a taste for migration). Immigrants have chosen to incur the migration costs already to move from their native country to the host country and therefore will have characteristics that reduces the cost of migration relative to other individuals in their country of origin. Hence, this self-selected group may also be the group of individuals with very low migration costs with regards to internal migration, relative to the same cost for natives.

In terms of the model laid out below, the first reason mentioned in the preceding paragraph would mean that the utility that an immigrant gains is not as much affected by the specific region/federal state the individual lives in as it is for natives. In other words, immigrants and natives differ with respect to the strength of their tastes for living in certain regions, which gives rise to non-monetary incentives to migrate. The second reason provided in the preceding paragraph would mean that unobservable characteristics that systematically differ between natives and immigrants yield the observed patterns.

Data from the German Socio-Economic Panel (GSOEP) allow me to provide some suggestive evidence related to the attachment to the region of current residence. In the GSOEP survey round of 2000, individuals were asked "To what extent do you feel connected to the place and area that you live in?". Table 6 below presents the responses of individuals who are more than 20 years of age. GSOEP distinguishes between foreigners and Germans based on citizenship. The table demonstrates that Germans are more than twice as likely to have "very strong" ties to the place and area they live in $(27.6 \%$ of natives have "very strong" ties, while only $11.8 \%$ of foreigners have "very strong" ties). On the other hand, foreigners are 
much more likely to respond that they are "not much" or "not at all" connected to the place and area they live in.

\begin{tabular}{lcc}
\hline \hline Do you have ties to the local area? & & \\
& Foreigner & Natives \\
\hline Very strong & $11.8 \%$ & $27.6 \%$ \\
Strong & $50.1 \%$ & $50.8 \%$ \\
Not much & $33.3 \%$ & $18.9 \%$ \\
Not at all & & \\
\hline & $4.8 \%$ & $2.7 \%$ \\
\hline observations & $100 \%$ & $100 \%$ \\
\hline Source: GSOEP data & 1,386 & 10,353 \\
\hline
\end{tabular}

Table 6: Ties to local area?

Thus, it appears likely that foreigners, on average, indeed derive less utility from the place and area they currently live in. Consequently, they should be more inclined to move away from that place. A question from GSOEP for the same individuals can shed additional light on this issue. Individuals were asked: "Would you consider moving away, e.g. because of family or job related reasons?". Table 7 summarizes the responses, again for those individuals of age 20 or more. The table shows that $32.6 \%$ of foreigners would consider moving away, while only $22.5 \%$ of natives would consider doing so. $46 \%$ of natives would not consider moving away, while $42 \%$ of foreigners would not consider moving away.

\begin{tabular}{|c|c|c|}
\hline \multicolumn{3}{|c|}{ Would you move away due to family or job related reasons? } \\
\hline & Foreigner & Natives \\
\hline yes & $32.6 \%$ & $22.5 \%$ \\
\hline possibly & $25.3 \%$ & $31.5 \%$ \\
\hline no & $42.0 \%$ & $46.0 \%$ \\
\hline & $100 \%$ & $100 \%$ \\
\hline observations & 1,385 & 10,350 \\
\hline
\end{tabular}

Source: GSOEP

Table 7: Would you move away?

Unfortunately, this direct survey evidence does not allow us to disentangle how important family reasons are versus job reasons. To learn more about the differences between natives and foreigners in their responsiveness to labor market conditions, I next resort to a structural econometric analysis of the migration decisions. 


\section{A structural model of migration decisions}

To be able to model the determinants of migration decisions explicitly, and to be able to quantify the differences between immigrants and natives in responsiveness to labor market conditions as well as differences in the unobserved cost of migration, I consider now an explicit structural model of migration decisions. As a consequence of the last section, namely the conclusion that economic as well as potentially unobserved, non-economic criteria determine the decision to migrate, I model this decision based on a comparison of utility levels attainable in different states and destinations. Assume that for individual $i$ the utility of living in destination $d$ is given by

$$
U_{d}^{i}=\beta X_{d}^{i}+\epsilon_{d}^{i}
$$

where $X_{d}^{i}$ are specific characteristics of choice $d$ for an individual $i$, and $\epsilon_{d}^{i}$ is an individual specific component of utility in destination $d .{ }^{15}$ Further assume that the error term $\epsilon_{d}^{i}$ is drawn i.i.d. from the Weibull distribution. This assumption gives us the conditional logit model. The utility maximizing individual $i$ will choose destination $d$ such that: $U_{d}^{i} \geq U_{j}^{i}$ for all $j \in\{1, \ldots, J\}$, where $J$ is the total number of destinations. In the present setup, $J$ will be equal to 17. This is because an individual living in state $o$ (origin) can choose among the following potential destinations: First, the individual could choose to migrate to one of the 15 other German federal states (there are 16 German federal states). Second, the individual could decide to stay within state of origin, $o$. In this case, there are two choices (destinations): either not to move at all, or move within the state of origin, o. Thus, there are a total of 17 different choices. Given the distributional assumption for the errors $\epsilon_{d}^{i}$, the probability that an individual $i$ chooses destination $d$ is

$$
\operatorname{prob}\left(U_{d}^{i} \geq U_{j}^{i}, \forall j \neq d\right)=\frac{e^{X_{d}^{i}}}{\sum_{h=1}^{J} e^{X_{h}^{i}}} .
$$

The parameters of the model can be estimated via maximum likelihood.

More specifically, assume that the utility that an individual $i$ gets by choosing to migrate to destination $d$ can be expressed as:

$$
\begin{aligned}
U_{d}^{i}= & \widetilde{\beta} \widetilde{X}_{d}^{i}+\gamma_{1} \text { distance }_{d}^{i}+\gamma_{2}\left(\text { distance }_{d}^{i}\right)^{2}+ \\
& +\delta \cdot I\{\text { migrating within state }\}+\eta \cdot I\{\text { migrating across state }\}+\epsilon_{d}^{i}
\end{aligned}
$$

\footnotetext{
${ }^{15}$ Note that we are considering internal migration and the individual already lives in a certain state. Consequently, destination characteristics may differ across individuals, depending on their current residence (the distance between state of current residence and destination state is one example), which is why $X_{d}^{i}$ is indexed with an $i$.
} 
where $\tilde{X}_{d}^{i}$ is a vector of destination characteristics, that are allowed to be specific to individual $i{ }^{16}$ distance $_{d}^{i}$ is the physical distance between the state of origin of individual $i$ and destination $d$, and $I$ \{migrating within state $\}$ and $I$ \{migrating across state $\}$ are two indicator functions. $I$ \{migrating within state $\}$ is equal to one for individual $i$ if the individual's choice of state is the state that he already resides in, but the choice implies that he moves within the state. $I$ \{migrating across state $\}$ is an indicator function which is equal to one for individual $i$ if the individual's choice of state is different from the state that he already resides in. While the previous discussion of the cost of migration has not distinguished between these two types of migration, one may expect that the cost of migration in fact differs depending on whether migration is within state or across state. The empirical setup allows for these two to differ, and the empirical work will show that indeed migration across state appears to be significantly more costly than migration within state. Because the importance of all variables may well differ between immigrants and natives, I will also estimate the conditional logit model separately for these two groups.

The key economic elements of interest in the vector of $\widetilde{X}_{d}^{i}$ are monthly income per capita (measured in units of 1,000 $\mathrm{DM}^{17}$ ) and the unemployment probability. In the few similar studies that I am aware of, which use aggregate data (e.g. Davies et al. 2001), these characteristics are assumed to be constant for all individuals in a state. Because of the large individual level data set, here I can significantly improve on that approach and take into account the heterogeneity of individuals, e.g. with respect to education and age. More specifically, I use the microcensus data to estimate wage and unemployment regressions separately for each state. The estimated parameters at the state level are then used to predict the income, conditional on being employed, and the probability of being unemployed for each individual in each state given the individual's characteristics. To predict income and unemployment I use different specifications, as will be explained below, and results are robust.

The distance variable is measured as the shortest road distance between the capital cities of the states (in 100 kilometers). This variable is included to capture the hypothesized changes in the cost of migration (for example due to cultural barriers, and information costs) if migration is to a state further away. Finally, there is a fundamental difference between moving and not moving. I capture this by the two dummy variables that indicate whether the individual moved over the last year, namely $I\{$ migrating within state $\}$ and $I$ \{migrating across state $\}$. Note that for the 17th destination, which is the hypothetical destination that implies migration

\footnotetext{
${ }^{16}$ The tilde above $\beta$ and $X$ is used to indicate that these are different $\beta$ and $X$ than the ones used in equation 1.

${ }^{17} \mathrm{DM}$ values are deflated to base year 2000 and Euro values in later survey years are expressed in DM. (For this base year, $1 \mathrm{DM}$ is approximately 0.50 USD.)
} 
within a state, all characteristics, such as unemployment rate or expected income, are the same as for the state of origin. Distance between the origin and this destination is zero.

The key interest is in the role of variables that vary at the individual level. To capture observable and unobservable characteristics that do not vary across individuals who choose a given state, I also add to most specifications (destination-) state fixed effects. These pick up, for example, variables related to the size of the state (either in terms of population or area), which are likely to have an impact on the probability of choosing a state. ${ }^{18}$ Note that the state fixed effects also control for unobserved state-level amenities, cultural differences, as well as existing immigrant concentrations within a state. ${ }^{19}$ The omitted state is SchleswigHolstein (the northernmost state of Germany). In some specifications I also use an West-East dummy, which is equal to one if an individual migrates from a western state to an eastern state or Berlin. The reason for investigating this separately is that there has been a fairly large return migration into federal states that were part of the former German Democratic Republic beginning in the mid 1990s (see e.g. Hunt 2006). This migration may not be explained by other observable characteristics, such as income or unemployment, but may rather be due to time and place specific circumstances after German Reunification.

\section{Income and unemployment prediction}

As mentioned above, I predict the income that an individual would be able to get (conditional on being employed) and the unemployment probability for each individual for each state. This approach allows for heterogeneity of income and unemployment in the conditional logit. To this end, I use Mincer-type regressions and regress, respectively, the logarithm of income and an indicator for whether an individual is unemployed, on a number of individual level characteristics. ${ }^{20}$ For this purpose, I use only employed individuals who are not working part-time, with positive recorded income, for whom wage income is the main income source (but predict income and unemployment for all individuals who remain in the sample for the conditional logit analysis).

Native and immigrant specific estimates are obtained by estimating income and unemployment regressions separately for immigrants and natives for each state. I have confirmed

\footnotetext{
${ }^{18}$ For example, if individuals pick a state randomly, with probabilities proportional to population size, more individuals would migrate to larger states.

${ }^{19}$ Note again that these state-fixed effects are also allowed to differ between immigrants and natives when I estimate the conditional logit model separately for these two groups.

${ }^{20}$ In particular, I include age, age squared, an indicator whether the individual is male or female, indicators for whether the individual has a higher schooling degree (at the level of the so-called "Abitur" or "Fachhochschulreife"), whether the individual has a lower level schooling degree (at the level of the "Hauptschule" or "Realschule" or "Oberschule"), lower vocational training ("Praktikum" or "Lehre" or "Berufsfachschule"), higher vocational training ("Meister" or "Fachschule") or a tertiary degree.
} 
that results do not change in important ways when I use an alternative specification in which I still estimate income and unemployment regressions separately for each state, but simply including a dummy variable to capture differences between immigrants and natives.

Using the estimates from the wage and unemployment regressions, I predict for all individuals in the sample (i.e. not just those in that state) what their income and their unemployment probability in a state would be. ${ }^{21}$

\section{Conditional logit estimation results}

\subsection{Determinants of internal migration in the total population}

This section presents the structural estimates of the parameters that determine migration decisions according to the model spelled out in equation 2. I use the same sample selection criteria that I employed for the probit models (mainly this implies that I restrict the analysis to 18-60 year olds who are either natives or immigrants, according to the above definitions, and who are not currently in school). Computational constraints require that I cannot work with the full sample that remains. ${ }^{22}$ Instead, I draw a $15 \%$ random subsample for estimation of the conditional logit models when I look at the pooled sample of natives and immigrants. This still leaves me with a sample size of 179,334 individuals. For the estimates based on only the immigrant (or recent immigrant) subsample, I can make use of the full (100\%) sample, which increases the precision of the estimates. Finally, for the analysis with the "household head/labor market participants" sample, which restricts the sample to household heads that are labor market participants, I am able to use a 30\% sample from the microcensus. In each table, I note which random sample is used. ${ }^{23}$

The results of the baseline regressions using the pooled data for natives plus immigrants

\footnotetext{
${ }^{21}$ One may be concerned that individuals might not move to a state at all if they do not have employment secured before they move, but (a) this could be relevant for households as only one member of the household may have secured employment before migrating, and (b) unemployment probabilities are indicators of future probabilities of unemployment, even if an individual is currently employed.

${ }^{22}$ The computational problem that prevents me from using the full sample of more than one million individual observations that I used in the probit analysis above is that the data set increases in size by a factor of 17 for the conditional logit estimation, because for each individual the dataset now adds an observation for each state. This requires large amounts of computer memory. Because of confidentiality requirements I am only able to access and work with the data remotely, and computational constraints at the German Federal Statistical Office could not be overcome.

${ }^{23}$ One may be worried that working with different random samples may affect the comparability of the results. Therefore, I have also estimated all conditional logit models with the same $15 \%$ random sample, which is the maximum that I can use for all samples, to confirm that the point estimates do not change more than would be expected based on the standard errors. Indeed, using the $15 \%$ sample throughout, implies less precise estimates for the immigrant and recent immigrant samples, as would be expected, but otherwise does not substantially change the qualitative picture.
} 
are reported in table 8. The estimates have the expected signs: A higher unemployment probability in a state reduces the probability that an individual chooses that state, while higher expected income in a destination increases the probability of choosing that destination. The probability of choosing a certain state decreases with the distance between the state of origin and the potential destination. The migration dummies are large in absolute size and highly significant. This indicates that, even taking into account income, unemployment and distance, there is still a large negative effect that is simply due to the mere fact that someone has to move (i.e. even if the destination of that move would have the same predicted income and unemployment and would be - a hypothetical - zero kilometers away). The West-East dummy that is used in the specification of column (3) is insignificant and I omit it in the later specifications. I investigate the robustness of the baseline results to using expected income (column 4), which is calculated as predicted income (i.e. income conditional on being employed) times (one minus the unemployment probability). The results are also robust to leaving out the unemployment probability in the model with expected income (column 5), using a sample with only singles (column 6) or using only heads of a household (column 7). 


\begin{tabular}{|c|c|c|c|c|c|c|c|}
\hline & baseline & $\begin{array}{l}\text { add state } \\
\text { effects } \\
\text { (2) }\end{array}$ & $\begin{array}{l}\text { add } \\
\text { West-East } \\
\text { indicator } \\
\text { (3) }\end{array}$ & $\begin{array}{l}\text { using } \\
\text { expected } \\
\text { income } \\
(4)\end{array}$ & $\begin{array}{l}\text { using } \\
\text { expected } \\
\text { income } \\
(5)\end{array}$ & $\begin{array}{l}\text { only } \\
\text { singles } \\
\text { (6) }\end{array}$ & $\begin{array}{l}\text { only head } \\
\text { of } \\
\text { household } \\
\text { (7) }\end{array}$ \\
\hline predicted destination income & $\begin{array}{l}0.364 \\
(0.049) * *\end{array}$ & $\begin{array}{l}0.311 \\
(0.051)^{* *}\end{array}$ & $\begin{array}{l}0.304 \\
(0.052)^{* *}\end{array}$ & & & $\begin{array}{l}0.378 \\
(0.090)^{* *}\end{array}$ & $\begin{array}{l}0.271 \\
(0.057) * *\end{array}$ \\
\hline $\begin{array}{l}\text { (predicted destination income)* } \\
\text { (1-probability of unemployment) }\end{array}$ & & & & $\begin{array}{l}0.330 \\
(0.051)^{* *}\end{array}$ & $\begin{array}{l}0.501 \\
(0.036)^{* *}\end{array}$ & & \\
\hline probability of unemployment & $\begin{array}{l}-2.593 \\
(0.272)^{* *}\end{array}$ & $\begin{array}{l}-2.149 \\
(0.282) * *\end{array}$ & $\begin{array}{l}-2.111 \\
(0.289) * *\end{array}$ & $\begin{array}{l}-1.573 \\
(0.335)^{* *}\end{array}$ & & $\begin{array}{l}-2.556 \\
(0.494) * *\end{array}$ & $\begin{array}{l}-2.187 \\
(0.411)^{* *}\end{array}$ \\
\hline migrate within state & $\begin{array}{l}-2.694 \\
(0.010)^{* *}\end{array}$ & $\begin{array}{l}-2.437 \\
(0.042)^{* *}\end{array}$ & $\begin{array}{l}-2.436 \\
(0.042)^{* *}\end{array}$ & $\begin{array}{l}-2.438 \\
(0.042)^{* *}\end{array}$ & $\begin{array}{l}-2.443 \\
(0.042)^{* *}\end{array}$ & $\begin{array}{l}-1.794 \\
(0.067)^{* *}\end{array}$ & $\begin{array}{l}-2.356 \\
(0.053) * *\end{array}$ \\
\hline migrate cross-state & $\begin{array}{l}-4.081 \\
(0.037)^{* *}\end{array}$ & $\begin{array}{l}-4.026 \\
(0.037)^{* *}\end{array}$ & $\begin{array}{l}-4.028 \\
(0.037)^{* *}\end{array}$ & $\begin{array}{l}-4.025 \\
(0.037) * *\end{array}$ & $\begin{array}{l}-4.028 \\
(0.037)^{* *}\end{array}$ & $\begin{array}{l}-3.510 \\
(0.059) * *\end{array}$ & $\begin{array}{l}-3.956 \\
(0.048) * *\end{array}$ \\
\hline distance & $\begin{array}{l}-1.619 \\
(0.029) * *\end{array}$ & $\begin{array}{l}-1.621 \\
(0.029) * *\end{array}$ & $\begin{array}{l}-1.615 \\
(0.031)^{* *}\end{array}$ & $\begin{array}{l}-1.622 \\
(0.029) * *\end{array}$ & $\begin{array}{l}-1.618 \\
(0.029) * *\end{array}$ & $\begin{array}{l}-1.484 \\
(0.044) * *\end{array}$ & $\begin{array}{l}-1.577 \\
(0.038) * *\end{array}$ \\
\hline distance squared & $\begin{array}{l}0.150 \\
(0.004)^{* *}\end{array}$ & $\begin{array}{l}0.152 \\
(0.004)^{* *}\end{array}$ & $\begin{array}{l}0.152 \\
(0.004) * *\end{array}$ & $\begin{array}{l}0.152 \\
(0.004) * *\end{array}$ & $\begin{array}{l}0.152 \\
(0.004) * *\end{array}$ & $\begin{array}{l}0.137 \\
(0.006)^{* *}\end{array}$ & $\begin{array}{l}0.147 \\
(0.005)^{* *}\end{array}$ \\
\hline Hamburg & & $\begin{array}{l}-0.126 \\
(0.061)^{*}\end{array}$ & $\begin{array}{l}-0.126 \\
(0.061)^{*}\end{array}$ & $\begin{array}{l}-0.123 \\
(0.061)^{*}\end{array}$ & $\begin{array}{l}-0.131 \\
(0.061)^{*}\end{array}$ & $\begin{array}{l}0.061 \\
(0.092)\end{array}$ & $\begin{array}{l}-0.025 \\
(0.077)\end{array}$ \\
\hline Niedersachsen & & $\begin{array}{l}0.395 \\
(0.050)^{* * *}\end{array}$ & $\begin{array}{l}0.395 \\
(0.050)^{* *}\end{array}$ & $\begin{array}{l}0.394 \\
(0.050)^{* *}\end{array}$ & $\begin{array}{l}0.395 \\
(0.050) * *\end{array}$ & $\begin{array}{l}0.375 \\
(0.080)^{* *}\end{array}$ & $\begin{array}{l}0.295 \\
(0.064)^{* *}\end{array}$ \\
\hline Bremen & & $\begin{array}{l}-0.196 \\
(0.064) * *\end{array}$ & $\begin{array}{l}-0.196 \\
(0.064)^{* *}\end{array}$ & $\begin{array}{l}-0.192 \\
(0.064) * *\end{array}$ & $\begin{array}{l}-0.195 \\
(0.064)^{* *}\end{array}$ & $\begin{array}{l}0.076 \\
(0.097)\end{array}$ & $\begin{array}{l}-0.155 \\
(0.080)\end{array}$ \\
\hline Nordrhein-Westfalen & & $\begin{array}{l}0.266 \\
(0.047)^{* *}\end{array}$ & $\begin{array}{l}0.267 \\
(0.047) * *\end{array}$ & $\begin{array}{l}0.266 \\
(0.047)^{* *}\end{array}$ & $\begin{array}{l}0.261 \\
(0.047)^{* *}\end{array}$ & $\begin{array}{l}0.373 \\
(0.075)^{* *}\end{array}$ & $\begin{array}{l}0.238 \\
(0.060) * *\end{array}$ \\
\hline Hessen & & $\begin{array}{l}0.263 \\
(0.050)^{* * *}\end{array}$ & $\begin{array}{l}0.264 \\
(0.050)^{* *}\end{array}$ & $\begin{array}{l}0.263 \\
(0.050)^{* *}\end{array}$ & $\begin{array}{l}0.258 \\
(0.050)^{* * *}\end{array}$ & $\begin{array}{l}0.363 \\
(0.080)^{* *}\end{array}$ & $\begin{array}{l}0.223 \\
(0.064)^{* *}\end{array}$ \\
\hline Rheinland-Pfalz & & $\begin{array}{l}0.172 \\
(0.051)^{* * *}\end{array}$ & $\begin{array}{l}0.173 \\
(0.051)^{* *}\end{array}$ & $\begin{array}{l}0.170 \\
(0.051)^{* * *}\end{array}$ & $\begin{array}{l}0.170 \\
(0.051)^{* *}\end{array}$ & $\begin{array}{l}0.232 \\
(0.082)^{* *}\end{array}$ & $\begin{array}{l}0.093 \\
(0.065)\end{array}$ \\
\hline Baden-Württemberg & & $\begin{array}{l}0.356 \\
(0.049)^{* *}\end{array}$ & $\begin{array}{l}0.357 \\
(0.049) * *\end{array}$ & $\begin{array}{l}0.354 \\
(0.049)^{* *}\end{array}$ & $\begin{array}{l}0.352 \\
(0.049) * *\end{array}$ & $\begin{array}{l}0.533 \\
(0.079) * *\end{array}$ & $\begin{array}{l}0.261 \\
(0.063)^{* *}\end{array}$ \\
\hline Bayern & & $\begin{array}{l}0.659 \\
(0.048)^{* * *}\end{array}$ & $\begin{array}{l}0.660 \\
(0.048)^{* *}\end{array}$ & $\begin{array}{l}0.657 \\
(0.048)^{* * *}\end{array}$ & $\begin{array}{l}0.656 \\
(0.048)^{* * *}\end{array}$ & $\begin{array}{l}0.795 \\
(0.076)^{* *}\end{array}$ & $\begin{array}{l}0.599 \\
(0.061)^{* *}\end{array}$ \\
\hline Saarland & & $\begin{array}{l}-0.081 \\
(0.065)\end{array}$ & $\begin{array}{l}-0.082 \\
(0.065)\end{array}$ & $\begin{array}{l}-0.078 \\
(0.065)\end{array}$ & $\begin{array}{l}-0.084 \\
(0.065)\end{array}$ & $\begin{array}{l}0.028 \\
(0.105)\end{array}$ & $\begin{array}{l}-0.128 \\
(0.083)\end{array}$ \\
\hline Berlin & & $\begin{array}{l}-0.120 \\
(0.054)^{*}\end{array}$ & $\begin{array}{l}-0.117 \\
(0.054)^{*}\end{array}$ & $\begin{array}{l}-0.115 \\
(0.054)^{*}\end{array}$ & $\begin{array}{l}-0.125 \\
(0.054)^{*}\end{array}$ & $\begin{array}{l}0.096 \\
(0.084)\end{array}$ & $\begin{array}{l}-0.050 \\
(0.068)\end{array}$ \\
\hline Brandenburg & & $\begin{array}{l}0.057 \\
(0.057)\end{array}$ & $\begin{array}{l}0.059 \\
(0.057)\end{array}$ & $\begin{array}{l}0.057 \\
(0.057)\end{array}$ & $\begin{array}{l}0.040 \\
(0.057)\end{array}$ & $\begin{array}{l}0.033 \\
(0.090)\end{array}$ & $\begin{array}{l}-0.061 \\
(0.073)\end{array}$ \\
\hline Mecklenburg-Vorpommern & & $\begin{array}{l}0.035 \\
(0.065)\end{array}$ & $\begin{array}{l}0.037 \\
(0.065)\end{array}$ & $\begin{array}{l}0.036 \\
(0.065)\end{array}$ & $\begin{array}{l}0.018 \\
(0.065)\end{array}$ & $\begin{array}{l}0.095 \\
(0.101)\end{array}$ & $\begin{array}{l}-0.046 \\
(0.083)\end{array}$ \\
\hline Sachsen & & $\begin{array}{l}-0.025 \\
(0.053)\end{array}$ & $\begin{array}{l}-0.024 \\
(0.053)\end{array}$ & $\begin{array}{l}-0.025 \\
(0.053)\end{array}$ & $\begin{array}{l}-0.032 \\
(0.053)\end{array}$ & $\begin{array}{l}-0.057 \\
(0.083)\end{array}$ & $\begin{array}{l}-0.133 \\
(0.067) *\end{array}$ \\
\hline Sachsen-Anhalt & & $\begin{array}{l}0.352 \\
(0.055)^{* * *}\end{array}$ & $\begin{array}{l}0.354 \\
(0.055) * *\end{array}$ & $\begin{array}{l}0.353 \\
(0.055)^{* *}\end{array}$ & $\begin{array}{l}0.325 \\
(0.054) * *\end{array}$ & $\begin{array}{l}0.417 \\
(0.087)^{* *}\end{array}$ & $\begin{array}{l}0.260 \\
(0.070)^{* *}\end{array}$ \\
\hline Thüringen & & $\begin{array}{l}0.175 \\
(0.061)^{* * *}\end{array}$ & $\begin{array}{l}0.177 \\
(0.061)^{* *}\end{array}$ & $\begin{array}{l}0.174 \\
(0.061)^{* * *}\end{array}$ & $\begin{array}{l}0.171 \\
(0.061)^{* *}\end{array}$ & $\begin{array}{l}0.203 \\
(0.098)^{*}\end{array}$ & $\begin{array}{l}0.082 \\
(0.079)\end{array}$ \\
\hline WestEast & & & $\begin{array}{l}-0.066 \\
(0.102) \\
\end{array}$ & & & & \\
\hline Observations & 179334 & 179334 & 179334 & 179334 & 179334 & 44605 & 97821 \\
\hline microcensus sample & $15 \%$ & $15 \%$ & $15 \%$ & $15 \%$ & $15 \%$ & $15 \%$ & $15 \%$ \\
\hline log-likelihood & -60062.4 & -59677.93 & -59677.72 & -59676.08 & -59687.13 & -22145.80 & -35877.79 \\
\hline
\end{tabular}

Notes: Standard errors in parentheses. * significant at 5\%; ** significant at 1\%.; omitted state is Schleswig-Holstein

Table 8: Conditional logit results: baseline results 


\subsection{The unobserved cost of migration}

Using the marginal rate of substitution between an attribute of a state and the income that an individual can obtain in that state given her characteristics, it is possible to assign a monetary value to the trade-offs between income and other relevant variables that affect the migration decision. For example, the trade-off between income and distance, i.e. the income change necessary to compensate for a change in the migration cost associated with the distance variable, can be calculated as follows (with $\gamma_{1}, \gamma_{2}$ and $\widetilde{\beta}_{\text {Income }}$ being the parameters on distance, distance squared, and income, respectively):

$$
\begin{gathered}
\frac{d \text { Income }}{d \text { Distance }}=-\frac{\frac{\partial U}{\partial \text { Distance }}}{\frac{\partial U}{\partial \text { Income }}} \\
\Leftrightarrow d \text { Income }=-\frac{\left(\gamma_{1}+2 \gamma_{2} \text { Distance }\right)}{\widetilde{\beta}_{\text {Income }}} d \text { Distance }
\end{gathered}
$$

Analogously, to calculate a measure of the unobserved costs of migration ${ }^{24}$, i.e. the disutility associated with migrating within a state (or leaving the state of origin), I calculate the change in annual income that is required to leave the probability of migrating within a state (or migrating to another state) unchanged. ${ }^{25}$ The unobserved cost of migration can therefore be calculated as follows

$$
\begin{array}{ll}
\text { Income }=\frac{\delta}{\widetilde{\beta}_{\text {Income }}} & \text { (migration within a state) } \\
\text { Income }=\frac{\eta}{\widetilde{\beta}_{\text {Income }}} \quad \text { (migration across a state) }
\end{array}
$$

where $\delta$ and $\eta$ are the parameter on the two migration indicator variables (see eq. 2 ). ${ }^{26}$

Using the parameter estimates from the baseline specification that includes state effects (column 2) in table 8, this implies, first, for the cost of distance: The (monthly) income change

\footnotetext{
${ }^{24}$ Note that one cannot term the "measure for the unobserved cost of migration" simply the "unobserved cost of migration". The income variable is annual income and the trade-off between not-moving and income will include discounted future income as well. Calculating the total unobserved cost would involve estimating the expected future income due to the migration. Nevertheless, the measure used here allows for an internally consistent comparison between natives and foreigners.

${ }^{25}$ Since the indicator variables are equal to one if the individual migrates (either within or across federal state borders), this number is negative and it can be interpreted as the amount of income that the individual would be willing to give for not having to move.

${ }^{26}$ Note that the coefficients reported are not marginal coefficients in the sense that they predict changes in choices in response to changes in covariates. However, they are marginal coefficients in the sense that they predict changes in utility in response to changes in covariates, which is what is required to perform the calculations that are proposed in this section.
} 
required to compensate for the first kilometer is about $52 \mathrm{DM}^{27}$, while at a distance of $100 \mathrm{~km}$ migrated already each additional kilometer of migration has to be compensated by 42 DM.

The estimated measure of the unobserved cost of migration to a different state, in terms of monthly income, is estimated to be 12,945 DM (in 2000 values, approximately US\$ 6,500), while the cost of migration within the state is estimated at 7,836 DM (approximately US\$ 3,900). This is the monetary incentive that an individual has to be given to make her migrate, everything else equal. This number may seem quite high but it is consistent with the overall very low migration rates (less than $2 \%$ for across-state migration in the data from the official registration records for the total population). Thus, the average cost of migration indeed needs to be fairly large to be able to explain these low migration rates in the presence of significant regional disparities across German states. Only few individuals will have idiosyncratic characteristics that influence the migration decision, i.e. in terms of the model draws of $\epsilon_{d}^{i}$, such that they reduce migration costs sufficiently to make internal migration attractive.

To judge these numbers, it may also be useful to compare them to the only other existing estimate that I am aware of. Davies et al. (2001) estimate the unobserved costs of cross-state migration in the US. However, they use a somewhat different empirical setup and the data they have available is different (in particular, they do not have micro data available for their work and they implicitly assume that income and unemployment rates are constant across all individuals in a state). These authors find unobserved cost of migration of between US\$ 170,000 to approximately US\$240,000 of (annual) per-capita income. Annualizing my findings, I get for cross-state migration a required income change of approximately US $\$ 6,500 \cdot 12=$ US\$ 78,000. Thus, the present findings are considerably lower, even after taking into account differences between the US and Germany in average annual incomes.

\subsection{Are immigrants more mobile than natives?}

I now turn to testing for differences between natives and immigrants. To test if there are differences between the internal migration behavior of natives and immigrants I first interact the key labor market variables with the immigrant indicator variable (columns 1 and 2 of table 9). Secondly, I run the earlier pooled regressions separately for natives and immigrants, as well as for recent immigrants (columns 3-5 of table 9).

\footnotetext{
${ }^{27}$ Recall that the income variable is measured in $1000 \mathrm{DM}$ and the distance variable is measured in $100 \mathrm{~km}$. Thus, to calculate the cost for the first kilometer moved: $(-(-1.621+2 \cdot 0.152 \cdot 0) / 0.311) \cdot 0.01=0.052$, i.e. 52 DM.
} 


\begin{tabular}{|c|c|c|c|c|c|}
\hline & $\begin{array}{l}\text { interactions } \\
\text { (1) }\end{array}$ & $\begin{array}{l}\text { interactions } \\
(2)\end{array}$ & $\begin{array}{l}\text { natives } \\
\text { (3) }\end{array}$ & $\begin{array}{l}\text { immigrants } \\
(4)\end{array}$ & $\begin{array}{l}\text { recent immigrants } \\
(5)\end{array}$ \\
\hline \multirow[t]{2}{*}{ predicted destination income } & 0.271 & 0.248 & 0.272 & 0.474 & 0.462 \\
\hline & $(0.054)^{* *}$ & $(0.055)^{* *}$ & $(0.055)^{* *}$ & $(0.064)^{* *}$ & $(0.069)^{* *}$ \\
\hline \multirow[t]{2}{*}{ probability of unemployment } & -2.053 & -1.974 & -2.032 & -2.335 & -2.394 \\
\hline & $(0.307)^{* *}$ & $(0.311)^{* *}$ & $(0.311)^{* *}$ & $(0.328)^{* *}$ & $(0.367)^{* *}$ \\
\hline \multirow[t]{2}{*}{ migrate within state } & -2.440 & -2.465 & -2.493 & -1.408 & -0.822 \\
\hline & $(0.042)^{* *}$ & $(0.042)^{* *}$ & $(0.044)^{* *}$ & $(0.059)^{* *}$ & $(0.074)^{* *}$ \\
\hline \multirow[t]{2}{*}{ migrate cross-state } & -4.022 & -4.181 & -4.180 & -2.726 & -2.059 \\
\hline & $(0.037)^{* *}$ & $(0.041)^{* *}$ & $(0.041)^{* *}$ & $(0.037)^{* *}$ & $(0.043)^{* *}$ \\
\hline \multirow[t]{2}{*}{ distance } & -1.620 & -1.542 & -1.545 & -2.017 & -2.068 \\
\hline & $(0.029)^{* *}$ & $(0.031)^{* *}$ & $(0.031)^{* *}$ & $(0.038)^{* *}$ & $(0.045)^{* *}$ \\
\hline \multirow[t]{2}{*}{ distance squared } & 0.152 & 0.144 & 0.144 & 0.195 & 0.201 \\
\hline & $(0.004)^{* *}$ & $(0.004)^{* *}$ & $(0.004)^{* *}$ & $(0.005)^{* *}$ & $(0.006)^{* *}$ \\
\hline \multirow{2}{*}{$\begin{array}{l}\text { immigrant* } \\
\text { (predicted destination income) }\end{array}$} & 0.456 & 0.512 & & & \\
\hline & $(0.156)^{* *}$ & $(0.143)^{* *}$ & & & \\
\hline \multirow{4}{*}{$\begin{array}{l}\text { immigrant* } \\
\text { (probability of unemployment) } \\
\text { immigrant*(migrate cross-state) }\end{array}$} & -1.292 & -1.198 & & & \\
\hline & $(0.832)$ & $(0.762)$ & & & \\
\hline & & 1.389 & & & \\
\hline & & $(0.104)^{* *}$ & & & \\
\hline \multirow[t]{2}{*}{ immigrant $*$ (migrate within state) } & & 0.635 & & & \\
\hline & & $(0.038)^{* *}$ & & & \\
\hline \multirow[t]{2}{*}{ immigrant*distance } & & -0.511 & & & \\
\hline & & $(0.102)^{* *}$ & & & \\
\hline \multirow[t]{2}{*}{ immigrant $*($ distance squared $)$} & & 0.055 & & & \\
\hline & & $(0.014)^{* *}$ & & & \\
\hline \multirow[t]{2}{*}{ Hamburg } & -0.124 & -0.091 & -0.128 & 0.278 & 0.262 \\
\hline & $(0.061)^{*}$ & $(0.061)$ & $(0.065)$ & $(0.074)^{* *}$ & $(0.092)^{* *}$ \\
\hline \multirow[t]{2}{*}{ Niedersachsen } & 0.396 & 0.397 & 0.384 & 0.484 & 0.597 \\
\hline & $(0.050)^{* *}$ & $(0.051)^{* *}$ & $(0.053)^{* *}$ & $(0.070)^{* *}$ & $(0.087)^{* *}$ \\
\hline \multirow[t]{2}{*}{ Bremen } & -0.198 & -0.178 & -0.201 & 0.103 & 0.122 \\
\hline & $(0.064)^{* *}$ & $(0.064)^{* *}$ & $(0.067)^{* *}$ & $(0.078)$ & $(0.097)$ \\
\hline \multirow[t]{2}{*}{ Nordrhein-Westfalen } & 0.264 & 0.283 & 0.241 & 0.805 & 0.932 \\
\hline & $(0.047)^{* *}$ & $(0.047)^{* *}$ & $(0.049)^{* *}$ & $(0.064)^{* *}$ & $(0.080)^{* *}$ \\
\hline \multirow[t]{2}{*}{ Hessen } & 0.259 & 0.288 & 0.241 & 0.759 & 0.916 \\
\hline & $(0.050)^{* *}$ & $(0.050)^{* *}$ & $(0.053)^{* *}$ & $(0.065)^{* *}$ & $(0.081)^{* *}$ \\
\hline \multirow[t]{2}{*}{ Rheinland-Pfalz } & 0.168 & 0.175 & 0.139 & 0.507 & 0.712 \\
\hline & $(0.051)^{* *}$ & $(0.051)^{* *}$ & $(0.053)^{* *}$ & $(0.067)^{* *}$ & $(0.083)^{* *}$ \\
\hline Baden-Württemberg & 0.353 & 0.390 & 0.328 & 0.936 & 1.151 \\
\hline & $(0.049)^{* *}$ & $(0.050)^{* *}$ & $(0.052)^{* *}$ & $(0.064)^{* *}$ & $(0.081)^{* *}$ \\
\hline Bayern & 0.654 & 0.670 & 0.648 & 1.122 & 1.327 \\
\hline & $(0.048)^{* *}$ & $(0.048) * *$ & $(0.050)^{* *}$ & $(0.064)^{* *}$ & $(0.080)^{* *}$ \\
\hline Saarland & -0.085 & -0.084 & -0.043 & -0.401 & -0.331 \\
\hline & $(0.065)$ & $(0.065)$ & $(0.068)$ & $(0.087)^{* *}$ & $(0.107)^{* *}$ \\
\hline Berlin & -0.118 & -0.095 & -0.166 & 0.575 & 0.830 \\
\hline & $(0.054)^{*}$ & $(0.054)$ & $(0.057)^{* *}$ & $(0.072)^{* *}$ & $(0.089)^{* *}$ \\
\hline Brandenburg & 0.054 & 0.036 & 0.031 & -0.410 & -0.187 \\
\hline & $(0.058)$ & $(0.058)$ & $(0.060)$ & $(0.108) * *$ & $(0.124)$ \\
\hline Mecklenburg-Vorpommern & 0.027 & 0.005 & 0.016 & -0.651 & -0.404 \\
\hline & $(0.065)$ & $(0.065)$ & $(0.068)$ & $(0.122)^{* *}$ & $(0.132)^{* *}$ \\
\hline Sachsen & -0.029 & -0.046 & -0.063 & -0.208 & 0.027 \\
\hline & $(0.053)$ & $(0.053)$ & $(0.055)$ & $(0.093) *$ & $(0.107)$ \\
\hline Sachsen-Anhalt & 0.347 & 0.326 & 0.322 & -0.024 & 0.272 \\
\hline & $(0.055)^{* *}$ & $(0.055)^{* *}$ & $(0.057)^{* *}$ & $(0.097)$ & $(0.108)^{*}$ \\
\hline Thüringen & 0.172 & 0.152 & 0.171 & -0.875 & -0.716 \\
\hline & $(0.061)^{* *}$ & $(0.062)^{*}$ & $(0.064)^{* *}$ & $(0.129)^{* *}$ & $(0.147)^{* *}$ \\
\hline Observations & 179334 & 179334 & 170742 & 57576 & 24249 \\
\hline microcensus sample & $15 \%$ & $15 \%$ & $15 \%$ & $100 \%$ & $100 \%$ \\
\hline log-likelihood & -59668.21 & -59491.62 & -54611.54 & -31824.2 & -19933.7 \\
\hline
\end{tabular}

Notes: Standard errors in parentheses. * significant at 5\%; ** significant at 1\%; omitted state is Schleswig-Holstein.

Table 9: Conditional logit results: natives and immigrants 
The results show some striking and statistically significant differences between natives and immigrants. First, consider columns (1) and (2) of table 9, in which I only allow the coefficients on predicted income and unemployment, as well as the distance and migration variables to vary with immigrant status, and restrict the state dummies to be the same across the two groups. Here I find that the response of immigrants to differences in per capita income between states is larger than the response of natives. Further, the coefficient on the destination unemployment rate is larger in absolute value for immigrants, suggesting that immigrants in Germany are more likely to respond to unemployment differentials than natives. Finally, the coefficient on the migration dummy variables (migrating within/across state borders) is larger in absolute terms for natives than for immigrants. All interaction terms except the interaction of immigrant with unemployment rate are statistically significant. ${ }^{28}$ Moving to the conditional logit results estimated separately for natives and immigrants, I find again that the coefficient on the migration dummy variables are larger in absolute terms for natives than for immigrants, indicating that natives are less likely to move at all. Further, the coefficients on the unemployment variable again indicate that immigrants are more likely to respond to unemployment differentials than natives. The coefficients for the income variable is significantly larger in the immigrant sample. ${ }^{29}$

One might expect labor market variables to be even more important once we focus exclusively on labor market participants. Therefore, I now analyze the "household head/labor market participants" sample that was introduced before, which in particular excludes members of the sample households that are not participating in the labor market or working part time. For this sample I also keep only the head of the household and drop individuals for which wage income is not the main source of income. Because these sample restrictions reduce the number of observations significantly, I can now use a larger sample from the full microcensus. For the analysis with the "household head/labor market participants" sample I am able to use a 30\% sample from the microcensus, which in particular increases substantially the precision of the estimates in the separate estimation of the conditional logit models for immigrants and recent immigrant. The results, which are reported in table 10, show the following: In all specifications and samples the indicator variables for cross-state and within-state migration are smaller in absolute size, and the coefficient on the unemployment variable is larger in absolute size than before. This suggests that overall the propensity to migrate and the reaction to

\footnotetext{
${ }^{28}$ The p-value for the parameter estimate on immigrant*(probability of unemployment) is 0.11 .

${ }^{29}$ While not all differences between individual coefficients are statistically significant, the joint statistical significance of the differences in the separate regressions is tested and confirmed using a Chow-test. The $H_{0}$ : coefficients are equal, is strongly rejected, with p-values $<0.0001$ for all three two-way comparisons, i.e. natives compared to immigrants, natives compared to recent immigrants, and all immigrants compared to the group of recent immigrants.
} 
differences in labor market characteristics is larger in this more narrowly defined sample. The only exception to this is the coefficient on the income variable, which is now smaller for the sample of natives than it was in the previous regressions. The results further indicate that the main findings regarding the differences between natives and immigrants are robust. In fact, for the unemployment and income variables the absolute difference between the coefficients for natives and immigrants increases compared to the results using the broader sample. 


\begin{tabular}{|c|c|c|c|c|c|}
\hline & $\begin{array}{l}\text { baseline } \\
(1)\end{array}$ & $\begin{array}{l}\text { interactions } \\
\text { (2) }\end{array}$ & $\begin{array}{l}\text { natives } \\
(3)\end{array}$ & $\begin{array}{l}\text { immigrants } \\
\text { (4) }\end{array}$ & $\begin{array}{l}\text { recent immigrants } \\
\text { (5) }\end{array}$ \\
\hline predicted destination income & $\begin{array}{l}0.207 \\
(0.044) * *\end{array}$ & $\begin{array}{l}0.152 \\
(0.047) * *\end{array}$ & $\begin{array}{l}0.164 \\
(0.047)^{* *}\end{array}$ & $\begin{array}{l}0.526 \\
(0.098) * *\end{array}$ & $\begin{array}{l}0.469 \\
(0.110)^{* *}\end{array}$ \\
\hline probability of unemployment & $\begin{array}{l}-2.955 \\
(0.341)^{* *}\end{array}$ & $\begin{array}{l}-2.817 \\
(0.368)^{* *}\end{array}$ & $\begin{array}{l}-2.835 \\
(0.369)^{* *}\end{array}$ & $\begin{array}{l}-3.548 \\
(0.583)^{* *}\end{array}$ & $\begin{array}{l}-3.538 \\
(0.714)^{* *}\end{array}$ \\
\hline migrate within state & $\begin{array}{l}-2.296 \\
(0.041)^{* *}\end{array}$ & $\begin{array}{l}-2.317 \\
(0.042)^{* *}\end{array}$ & $\begin{array}{l}-2.328 \\
(0.043)^{* *}\end{array}$ & $\begin{array}{l}-1.368 \\
(0.099)^{* *}\end{array}$ & $\begin{array}{l}-0.693 \\
(0.132)^{* *}\end{array}$ \\
\hline migrate cross-state & $\begin{array}{l}-3.996 \\
(0.038)^{* *}\end{array}$ & $\begin{array}{l}-4.082 \\
(0.040)^{* *}\end{array}$ & $\begin{array}{l}-4.081 \\
(0.040)^{* *}\end{array}$ & $\begin{array}{l}-2.938 \\
(0.061)^{* *}\end{array}$ & $\begin{array}{l}-2.145 \\
(0.076)^{* *}\end{array}$ \\
\hline distance & $\begin{array}{l}-1.532 \\
(0.029)^{* *}\end{array}$ & $\begin{array}{l}-1.489 \\
(0.030)^{* *}\end{array}$ & $\begin{array}{l}-1.490 \\
(0.030)^{* *}\end{array}$ & $\begin{array}{l}-1.877 \\
(0.058)^{* *}\end{array}$ & $\begin{array}{l}-1.897 \\
(0.070)^{* *}\end{array}$ \\
\hline distance squared & $\begin{array}{l}0.144 \\
(0.004)^{* *}\end{array}$ & $\begin{array}{l}0.139 \\
(0.004)^{* *}\end{array}$ & $\begin{array}{l}0.139 \\
(0.004) * *\end{array}$ & $\begin{array}{l}0.183 \\
(0.008) * *\end{array}$ & $\begin{array}{l}0.186 \\
(0.010)^{* *}\end{array}$ \\
\hline $\begin{array}{l}\text { immigrant* } \\
\text { (predicted destination income) }\end{array}$ & & $\begin{array}{l}0.782 \\
(0.158) * *\end{array}$ & & & \\
\hline $\begin{array}{l}\text { immigrant* } \\
\text { (probability of unemployment) }\end{array}$ & & $\begin{array}{l}-1.052 \\
(0.992)\end{array}$ & & & \\
\hline immigrant*(migrate cross-state) & & $\begin{array}{l}1.052 \\
(0.120)^{* *}\end{array}$ & & & \\
\hline immigrant*(migrate within state) & & $\begin{array}{l}0.503 \\
(0.040)^{* *}\end{array}$ & & & \\
\hline immigrant*(distance) & & $\begin{array}{l}-0.378 \\
(0.109)^{* *}\end{array}$ & & & \\
\hline immigrant $*($ distance squared $)$ & & $\begin{array}{l}0.041 \\
(0.015)^{* *}\end{array}$ & & & \\
\hline Hamburg & $\begin{array}{l}-0.032 \\
(0.060)\end{array}$ & $\begin{array}{l}-0.007 \\
(0.060)\end{array}$ & $\begin{array}{l}-0.021 \\
(0.063)\end{array}$ & $\begin{array}{l}0.328 \\
(0.123)^{* *}\end{array}$ & $\begin{array}{l}0.286 \\
(0.163)\end{array}$ \\
\hline Niedersachsen & $\begin{array}{l}0.363 \\
(0.050) * *\end{array}$ & $\begin{array}{l}0.367 \\
(0.050) * *\end{array}$ & $\begin{array}{l}0.357 \\
(0.051)^{* *}\end{array}$ & $\begin{array}{l}0.538 \\
(0.116) * *\end{array}$ & $\begin{array}{l}0.685 \\
(0.153) * *\end{array}$ \\
\hline Bremen & $\begin{array}{l}-0.082 \\
(0.062)\end{array}$ & $\begin{array}{l}-0.070 \\
(0.062)\end{array}$ & $\begin{array}{l}-0.084 \\
(0.065)\end{array}$ & $\begin{array}{l}0.371 \\
(0.129)^{* *}\end{array}$ & $\begin{array}{l}0.345 \\
(0.171)^{*}\end{array}$ \\
\hline Nordrhein-Westfalen & $\begin{array}{l}0.232 \\
(0.046)^{* *}\end{array}$ & $\begin{array}{l}0.243 \\
(0.046)^{* *}\end{array}$ & $\begin{array}{l}0.224 \\
(0.048) * *\end{array}$ & $\begin{array}{l}0.730 \\
(0.106) * *\end{array}$ & $\begin{array}{l}0.800 \\
(0.143)^{* *}\end{array}$ \\
\hline Hessen & $\begin{array}{l}0.235 \\
(0.049)^{* *}\end{array}$ & $\begin{array}{l}0.252 \\
(0.049)^{* *}\end{array}$ & $\begin{array}{l}0.219 \\
(0.051)^{* *}\end{array}$ & $\begin{array}{l}0.815 \\
(0.108)^{* *}\end{array}$ & $\begin{array}{l}1.041 \\
(0.144)^{* *}\end{array}$ \\
\hline Rheinland-Pfalz & $\begin{array}{l}0.129 \\
(0.050)^{*}\end{array}$ & $\begin{array}{l}0.133 \\
(0.050)^{* *}\end{array}$ & $\begin{array}{l}0.129 \\
(0.052)^{*}\end{array}$ & $\begin{array}{l}0.477 \\
(0.112)^{* *}\end{array}$ & $\begin{array}{l}0.708 \\
(0.148)^{* *}\end{array}$ \\
\hline Baden-Württemberg & $\begin{array}{l}0.300 \\
(0.048)^{* *}\end{array}$ & $\begin{array}{l}0.327 \\
(0.049)^{* *}\end{array}$ & $\begin{array}{l}0.317 \\
(0.051)^{* *}\end{array}$ & $\begin{array}{l}0.850 \\
(0.106) * *\end{array}$ & $\begin{array}{l}1.001 \\
(0.143)^{* *}\end{array}$ \\
\hline Bayern & $\begin{array}{l}0.636 \\
(0.047)^{* *}\end{array}$ & $\begin{array}{l}0.644 \\
(0.047)^{* *}\end{array}$ & $\begin{array}{l}0.634 \\
(0.049) * *\end{array}$ & $\begin{array}{l}1.152 \\
(0.106) * *\end{array}$ & $\begin{array}{l}1.373 \\
(0.140)^{* *}\end{array}$ \\
\hline Saarland & $\begin{array}{l}-0.150 \\
(0.064)^{*}\end{array}$ & $\begin{array}{l}-0.146 \\
(0.064)^{*}\end{array}$ & $\begin{array}{l}-0.135 \\
(0.066)^{*}\end{array}$ & $\begin{array}{l}-0.234 \\
(0.147)\end{array}$ & $\begin{array}{l}-0.169 \\
(0.195)\end{array}$ \\
\hline Berlin & $\begin{array}{l}-0.013 \\
(0.053)\end{array}$ & $\begin{array}{l}0.002 \\
(0.053)\end{array}$ & $\begin{array}{l}-0.038 \\
(0.055)\end{array}$ & $\begin{array}{l}0.600 \\
(0.121)^{* *}\end{array}$ & $\begin{array}{l}0.758 \\
(0.161)^{* *}\end{array}$ \\
\hline Brandenburg & $\begin{array}{l}0.049 \\
(0.057)\end{array}$ & $\begin{array}{l}0.029 \\
(0.057)\end{array}$ & $\begin{array}{l}0.028 \\
(0.058)\end{array}$ & $\begin{array}{l}-0.399 \\
(0.181)^{*}\end{array}$ & $\begin{array}{l}-0.183 \\
(0.218)\end{array}$ \\
\hline Mecklenburg-Vorpommern & $\begin{array}{l}0.062 \\
(0.064)\end{array}$ & $\begin{array}{l}0.036 \\
(0.064)\end{array}$ & $\begin{array}{l}0.053 \\
(0.066)\end{array}$ & $\begin{array}{l}-0.712 \\
(0.222)^{* *}\end{array}$ & $\begin{array}{l}-0.564 \\
(0.262)^{*}\end{array}$ \\
\hline Sachsen & $\begin{array}{l}-0.042 \\
(0.052)\end{array}$ & $\begin{array}{l}-0.059 \\
(0.052)\end{array}$ & $\begin{array}{l}-0.066 \\
(0.053)\end{array}$ & $\begin{array}{l}-0.044 \\
(0.158)\end{array}$ & $\begin{array}{l}0.095 \\
(0.195)\end{array}$ \\
\hline Sachsen-Anhalt & $\begin{array}{l}0.343 \\
(0.054)^{* *}\end{array}$ & $\begin{array}{l}0.322 \\
(0.054) * *\end{array}$ & $\begin{array}{l}0.327 \\
(0.056) * *\end{array}$ & $\begin{array}{l}0.148 \\
(0.172)\end{array}$ & $\begin{array}{l}0.439 \\
(0.201) *\end{array}$ \\
\hline Thüringen & $\begin{array}{l}0.149 \\
(0.061)^{*}\end{array}$ & $\begin{array}{l}0.130 \\
(0.062) *\end{array}$ & $\begin{array}{l}0.138 \\
(0.063) *\end{array}$ & $\begin{array}{l}-0.555 \\
(0.214)^{* *}\end{array}$ & $\begin{array}{l}-0.384 \\
(0.246)\end{array}$ \\
\hline $\begin{array}{l}\text { Observations } \\
\text { microcensus sample } \\
\text { log-likelihood }\end{array}$ & $\begin{array}{l}162699 \\
30 \% \\
-60185.4\end{array}$ & $\begin{array}{l}162699 \\
30 \% \\
-60072.0\end{array}$ & $\begin{array}{l}155344 \\
30 \% \\
-56211.1\end{array}$ & $\begin{array}{l}24523 \\
100 \% \\
-12766.4\end{array}$ & $\begin{array}{l}8457 \\
100 \% \\
-6811.0\end{array}$ \\
\hline
\end{tabular}

Notes: Standard errors in parentheses. * significant at 5\%; ** significant at 1\%; omitted state is Schleswig-Holstein.

Table 10: Conditional logit results, robustness checks with the "household head/labor market participants" sample (for details about the sample restrictions see text) 
In sum, the results of this subsection are consistent with the hypothesis that immigrants are more mobile with respect to internal mobility than natives. This difference, as measured through the migration dummy variables, is statistically significant. In addition, I also find that economic variables are stronger determinants of internal migration decisions for immigrants than for natives. There are economically significant differences between natives' and immigrants' reactions to income as well as unemployment differentials across states in all specifications. In the estimation by group (columns 3 and 4 ) we find statistically significant differences with respect to income. It is noteworthy that the finding that economic variables are significant determinants of internal migration decisions of immigrants is in contrast to Bartel and Koch (1991) for the US. ${ }^{30}$

\subsection{The unobserved cost of migration of natives and immigrants}

Following the same approach as before I measure the unobserved cost of migration using the estimates from columns (3), (4), and (5), respectively, of table 9. The results are summarized in table 11, which repeats the earlier results for the total population for comparison.

Table 11 summarizes the key results: cross-state and within-state migration is less costly for immigrants than for native Germans. The measure of unobserved cost of migration for cross state migration is estimated to be about 2.67 times larger for natives than for immigrants: for natives it is approximately 15,400 DM (US\$ 7,700) while for immigrants it is approximately $5,750 \mathrm{DM}$ (US\$2,875); that is, the measure of unobserved cost of migration for immigrants is $37 \%$ of the cost for natives. Recent immigrants show even lower cost of migration than the group of immigrants as a whole.

\footnotetext{
${ }^{30}$ A potentially important reason for this difference, in addition to the fact that Bartel and Koch (1991) study internal migration in the US, is that that these authors focus on push factors, i.e. conditions in the origin, while the present method compares the conditions in the origin to conditions in all potential destinations. They assume that the push factors such as wage and unemployment rates are constant for all individuals in a region of origin.
} 


\begin{tabular}{|c|c|c|}
\hline & \multicolumn{2}{|c|}{$\begin{array}{l}\text { measure of unobserved cost } \\
\text { of migration } \\
\text { (monthly income change, in DM) }\end{array}$} \\
\hline & within state & across state \\
\hline Total population & 7,836 & 12,945 \\
\hline Natives & 9,165 & 15,368 \\
\hline Immigrants & 2,970 & 5,751 \\
\hline Recent immigrants & 1,780 & 4,457 \\
\hline
\end{tabular}

Table 11: The estimated trade-offs for natives and foreigners

\subsection{Analysis by age}

As we have seen above, the age variables are both statistically and economically significant predictors of migration decisions in the reduced form probit analysis. To further investigate the differences in the propensity to migrate over the life cycle, and the role that labor market characteristics play for this, I re-estimate the baseline conditional logit regression separately for each age group. The analysis by age group is also motivated by recent findings by Hunt (2006). Hunt uses state-level data and GSOEP data to demonstrate that important differences in migration behavior between different age groups can help explain the puzzle that in aggregate data unemployment often does not predict migration behavior. By looking at different age groups separately, she finds that this is indeed only true for young cohorts, but not for the older cohorts. Here, I can use a much larger sample of individual level data to shed additional light on this question from a somewhat different angle. ${ }^{31}$ To the extent that these two sides of the migration decision can be separated, Hunt (2006) studies more the push factors, with a focus of conditions in their origin, while I study pull factors with a focus that is somewhat more on the question: what conditions in potential destinations attract individuals?

In line with previous findings for the push factors I find that the younger age groups (18-24 year and 25-34 year olds) are more responsive to income differentials than older individuals. I also find that 25-34 year olds have larger responsiveness to expected unemployment rates than the youngest individuals in the sample. On the other hand, I find that unemployment rates appear to be least important for migration decisions for the oldest age group (table 12).

\footnotetext{
${ }^{31}$ Note that I also split the age distribution more finely than Hunt does (she uses ages 18-24, 25-49, 50-64).
} 
Repeating this analysis by age separately for immigrants and natives reveals that the observed pattern regarding the role of income as a pull factor is not unique to natives (table 13). Immigrants, too, show a relatively larger response to income differentials across states when they are young, and smaller responsiveness when they are old. Both natives and immigrants have the lowest responsiveness to unemployment differentials in the older age groups. The parameter estimates on the migration related dummies are smaller in absolute size for immigrants across all age groups. Combined with the larger responsiveness to income differences this implies that for all age groups the unobserved cost of migration is smaller for immigrants. 


\begin{tabular}{|c|c|c|c|c|c|}
\hline & $\begin{array}{l}\text { all } \\
(1)\end{array}$ & $\begin{array}{l}18-24 \\
(2)\end{array}$ & $\begin{array}{l}25-34 \\
(3)\end{array}$ & $\begin{array}{l}35-44 \\
(4)\end{array}$ & $\begin{array}{l}>=45 \\
(5)\end{array}$ \\
\hline \multirow[t]{2}{*}{ predicted destination income } & 0.311 & 0.891 & 0.695 & 0.477 & 0.258 \\
\hline & $(0.051)^{* *}$ & $(0.254)^{* *}$ & $(0.109)^{* *}$ & $(0.103) * *$ & $(0.103)^{*}$ \\
\hline \multirow[t]{2}{*}{ probability of unemployment } & -2.149 & -3.023 & -3.495 & -2.321 & -0.972 \\
\hline & $(0.282)^{* *}$ & $(0.705)^{* *}$ & $(0.559)^{* *}$ & $(0.681)^{* *}$ & $(0.590)$ \\
\hline \multirow[t]{2}{*}{ migrate within state } & -2.437 & -1.336 & -1.798 & -2.481 & -3.479 \\
\hline & $(0.042)^{* *}$ & $(0.116)^{* *}$ & $(0.067)^{* *}$ & $(0.081)^{* *}$ & $(0.101)^{* *}$ \\
\hline \multirow[t]{2}{*}{ migrate cross-state } & -4.026 & -3.032 & -3.401 & -4.112 & -4.887 \\
\hline & $(0.037)^{* *}$ & $(0.094)^{* *}$ & $(0.058)^{* *}$ & $(0.072)^{* *}$ & $(0.089)^{* *}$ \\
\hline \multirow[t]{2}{*}{ distance } & -1.621 & -1.618 & -1.559 & -1.710 & -1.715 \\
\hline & $(0.029)^{* *}$ & $(0.072)^{* *}$ & $(0.045)^{* *}$ & $(0.059)^{* *}$ & $(0.073)^{* *}$ \\
\hline \multirow[t]{2}{*}{ distance squared } & 0.152 & 0.154 & 0.144 & 0.162 & 0.162 \\
\hline & $(0.004)^{* *}$ & $(0.010)^{* *}$ & $(0.006)^{* *}$ & $(0.008)^{* *}$ & $(0.010)^{* *}$ \\
\hline \multirow[t]{2}{*}{ Hamburg } & -0.126 & 0.054 & -0.127 & -0.076 & -0.239 \\
\hline & $(0.061)^{*}$ & $(0.167)$ & $(0.097)$ & $(0.121)$ & $(0.146)$ \\
\hline \multirow[t]{2}{*}{ Niedersachsen } & 0.395 & 0.404 & 0.394 & 0.514 & 0.312 \\
\hline & $(0.050)^{* *}$ & $(0.137)^{* *}$ & $(0.080)^{* *}$ & $(0.099) * *$ & $(0.121)^{* *}$ \\
\hline \multirow[t]{2}{*}{ Bremen } & -0.196 & 0.079 & -0.190 & -0.164 & -0.406 \\
\hline & $(0.064)^{* *}$ & $(0.177)$ & $(0.102)$ & $(0.124)$ & $(0.150)^{* *}$ \\
\hline \multirow[t]{2}{*}{ Nordrhein-Westfalen } & 0.266 & 0.464 & 0.237 & 0.310 & 0.174 \\
\hline & $(0.047)^{* *}$ & $(0.130)^{* *}$ & $(0.075)^{* *}$ & $(0.090)^{* *}$ & $(0.113)$ \\
\hline \multirow[t]{2}{*}{ Hessen } & 0.263 & 0.407 & 0.176 & 0.339 & 0.193 \\
\hline & $(0.050)^{* *}$ & $(0.138)^{* *}$ & $(0.079)^{*}$ & $(0.096)^{* *}$ & $(0.120)$ \\
\hline \multirow[t]{2}{*}{ Rheinland-Pfalz } & 0.172 & 0.303 & 0.111 & 0.321 & 0.026 \\
\hline & $(0.051)^{* *}$ & $(0.139)^{*}$ & $(0.081)$ & $(0.098)^{* *}$ & $(0.122)$ \\
\hline \multirow[t]{2}{*}{ Baden-Württemberg } & 0.356 & 0.474 & 0.450 & 0.360 & 0.191 \\
\hline & $(0.049)^{* *}$ & $(0.135)^{* *}$ & $(0.079) * *$ & $(0.095)^{* *}$ & $(0.119)$ \\
\hline \multirow[t]{2}{*}{ Bayern } & 0.659 & 0.726 & 0.650 & 0.829 & 0.659 \\
\hline & $(0.048) * *$ & $(0.128)^{* *}$ & $(0.076)^{* *}$ & $(0.093) * *$ & $(0.117)^{* *}$ \\
\hline \multirow[t]{2}{*}{ Saarland } & -0.081 & -0.078 & -0.214 & 0.007 & -0.132 \\
\hline & $(0.065)$ & $(0.182)$ & $(0.107)^{*}$ & $(0.127)$ & $(0.152)$ \\
\hline \multirow[t]{2}{*}{ Berlin } & -0.120 & 0.032 & -0.042 & -0.075 & -0.384 \\
\hline & $(0.054)^{*}$ & $(0.152)$ & $(0.088)$ & $(0.105)$ & $(0.127)^{* *}$ \\
\hline \multirow[t]{2}{*}{ Brandenburg } & 0.057 & -0.001 & 0.162 & 0.271 & -0.163 \\
\hline & $(0.057)$ & $(0.151)$ & $(0.094)$ & $(0.113)^{*}$ & $(0.137)$ \\
\hline \multirow[t]{2}{*}{ Mecklenburg-Vorpommern } & 0.035 & 0.302 & 0.202 & 0.121 & -0.176 \\
\hline & $(0.065)$ & $(0.164)$ & $(0.109)$ & $(0.129)$ & $(0.155)$ \\
\hline \multirow[t]{2}{*}{ Sachsen } & -0.025 & 0.057 & 0.087 & 0.058 & -0.282 \\
\hline & $(0.053)$ & $(0.140)$ & $(0.086)$ & $(0.103)$ & $(0.124)^{*}$ \\
\hline \multirow[t]{2}{*}{ Sachsen-Anhalt } & 0.352 & 0.434 & 0.560 & 0.447 & 0.017 \\
\hline & $(0.055)^{* *}$ & $(0.145)^{* *}$ & $(0.090)^{* *}$ & $(0.107)^{* *}$ & $(0.128)$ \\
\hline \multirow[t]{2}{*}{ Thüringen } & 0.175 & 0.317 & 0.309 & 0.270 & -0.086 \\
\hline & $(0.061)^{* *}$ & $(0.162)^{*}$ & $(0.101)^{* *}$ & $(0.120)^{*}$ & $(0.146)$ \\
\hline Observations & 179334 & 12090 & 42541 & 52429 & 72274 \\
\hline microcensus sample & $15 \%$ & $15 \%$ & $15 \%$ & $15 \%$ & $15 \%$ \\
\hline log-likelihood & -59677.93 & -7643.26 & -21466.60 & -15690.30 & -12099.69 \\
\hline
\end{tabular}

Notes: Standard errors in parentheses. * significant at 5\%; ** significant at 1\%; omitted state is Schleswig-Holstein.

Table 12: Conditional logit results: by age 


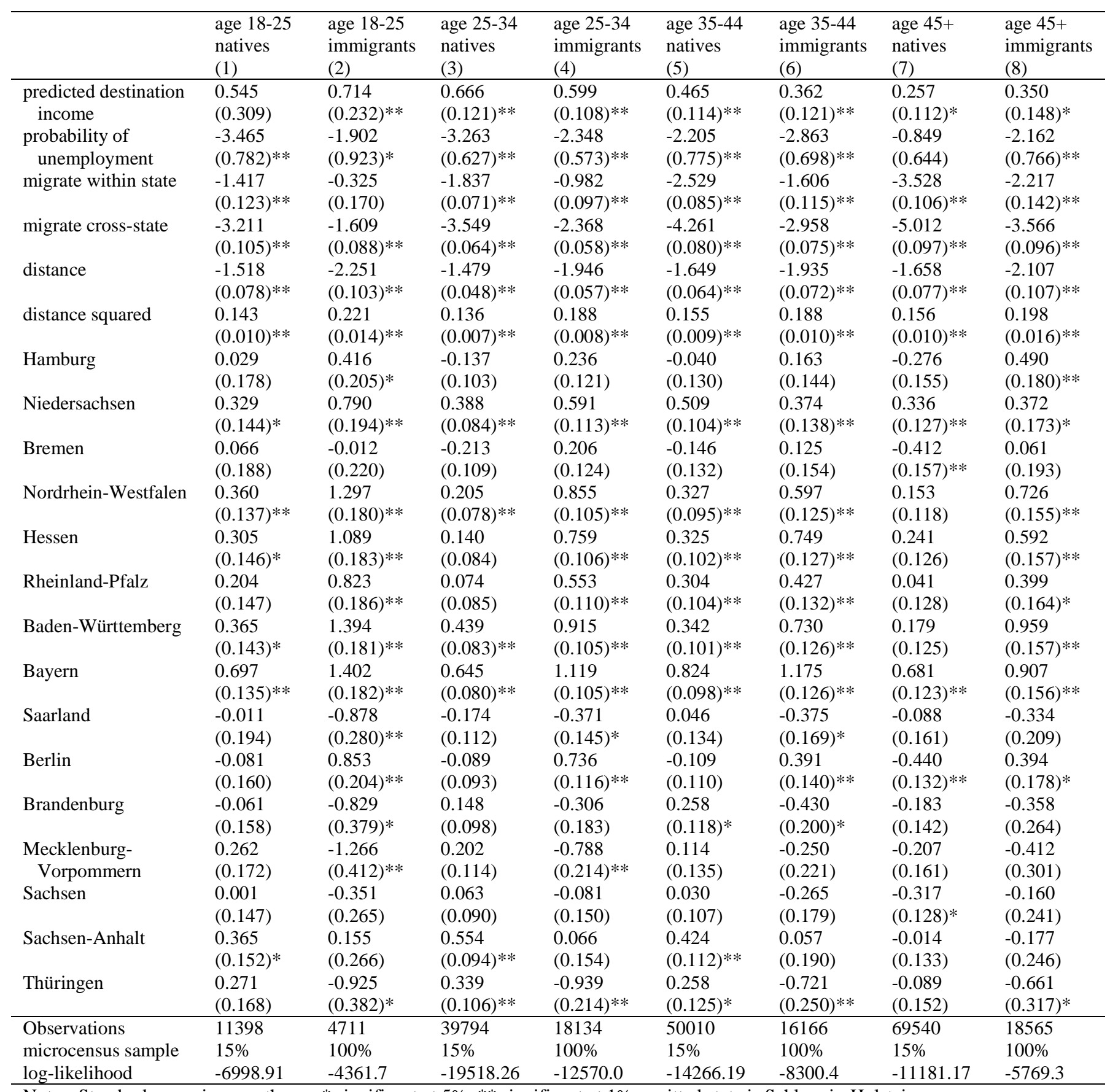

Notes: Standard errors in parentheses. * significant at 5\%; ** significant at 1\%; omitted state is Schleswig-Holstein.

Table 13: Conditional logit results: by age and separate for natives and immigrants 


\section{Conclusion}

Convergence of wage levels and of other labor market characteristics can yield substantial efficiency gains (e.g. Borjas 2001). However, it is known that flows of labor (and capital) do not respond to labor market differentials instantaneously (e.g. Barro and Sala-i-Martin 1992, Decressin and Fatás 1995). A major impediment to labor flows are migration costs, and it is important to understand the determinants and the magnitude of these migration costs for different subgroups of the population. Of particular policy relevance are differences between natives and immigrants.

In this paper, I show that immigrants are more likely than natives to migrate internally within Germany. This is true even after controlling for a large number of individual-specific characteristics. Thus, selection based on observable characteristics can explain some, but not all of the observed differences in the aggregate. I further present econometric evidence, based on a structural model of migration decisions, for significant differences in migration costs between natives and immigrants in Germany. Immigrants are significantly more responsive to labor market differentials than natives. Further, I estimate that the unobserved cost of migration for immigrants is only about $37 \%$ of the cost for natives. The findings of overall high migration costs are consistent with low migration rates in the population, while the observed differences between natives and immigrants confirm relatively unexplored conjectures in the literature. Using GSOEP-data I can provide some additional qualitative evidence that suggests that ties to the region in which immigrants live in Germany are weaker than the ties of natives to their region, which in turn suggests a potential explanation for the quantitative findings.

The findings bear on policy. In the presence of high unemployment rates, immigration is sometimes seen as placing an additional burden on the labor markets of receiving countries. However, in the case of Germany, the large migration-responsiveness of immigrants to labor market differentials can be expected to have positive macro effects as it increases the speed of convergence between regions and accelerates adjustments in response to regional shocks. Thus the results suggest that, in addition to wage and employment effects, as a third dimension internal migration should be studied to estimate the effects of immigration on a host country's labor market. The findings in this paper have implications for other countries with similar regional disparities across labor markets and significant presence of immigrants and are of relevance to all West European countries for an assessment of the economic impact of the immigrant flows that are expected in the wake of the EU expansion towards Eastern Europe. 


\section{References}

[1] Angrist, Joshua, and Adriana Kugler, 2001, Protective or Counter-Protective? Labor Market Institutions and the Effect of Immigration on EU Natives. NBER Working Paper 8660 .

[2] Appleyard, Reginald, ed., 1998, Emigration Dynamics in Developing Countries. Ashgate, Brookfield, VT.

[3] Barro, Robert, and Xavier Sala-i-Martin, 1992, Regional Growth and Migration: A Japan-US Comparison. NBER Working Paper 4038.

[4] Bartel, Ann, 1989, Where Do the New U.S. Immigrants Live? Journal of Labor Economics 7 (4), 371-391.

[5] Bartel, Ann, and Marianne Koch, 1991, Internal Migration of U.S. Immigrants. In: John Abowd and Richard Freeman, eds., Immigration, Trade, and the Labor Market. University of Chicago Press, Chicago, 121-134.

[6] Bauer, Thomas, and Klaus Zimmermann, 1999, Assessment of Possible Migration Pressure and its Labor Market Impact Following EU Enlargement to Central and Eastern Europe. IZA Research Report No. 3, Bonn.

[7] Blanchard, Olivier, and Lawrence Katz, 1992, Regional Evolutions. Brookings Papers on Economic Activity 1, 1-75.

[8] Borjas, George, 1987, Self-Selection and the Earnings of Immigrants. American Economic Review 77 (4), 531-553.

[9] Borjas, George, 1999a, Immigration and Welfare Magnets. Journal of Labor Economics $17(4), 607-637$.

[10] Borjas, George, 1999b, The Economic Analysis of Immigration. In: O. Ashenfelter and D. Card, eds., Handbook of Labor Economics, Vol. 3a, Chapter 28.

[11] Borjas, George, 2001, Does Immigration Grease the Wheels of the Labor Market? Brookings Papers on Economic Activity, 1, 69-133.

[12] Borjas, George, Stephen Bronars, and Stephen Trejo, 1992, Self-Selection and Internal Migration in the United States. Journal of Urban Economics 32 (2), 159-185. 
[13] Chiswick, Barry, 2000, Are Immigrants Favorably Self-Selected? An Economic Analysis, IZA Discussion Papers 131, Bonn.

[14] Davies, Paul, Michael J. Greenwood, and Haizheng Li, 2001, A Conditional Logit Approach to U.S. State-to-State Migration. Journal of Regional Science 41 (2), 337-360.

[15] Decressin, Jörg, 1994, Internal Migration in West Germany and Implications for EastWest Salary Convergence. Weltwirtschaftliches Archiv, 231-257.

[16] Decressin, Jörg, and Antonio Fatás, 1995, Regional Labor Market Dynamics in Europe. European Economic Review 39, 1627-1655.

[17] De New, John, and Klaus Zimmermann, 1994, Native Wage Impacts of Foreign Labor: A Random Effects Panel Analysis. Journal of Population Economics 7, 177-192.

[18] Dustmann, Christian, 2003, Return Migration, Wage Differentials, and the Optimal Migration Duration. European Economic Review, 47 (2), 353-369.

[19] Faini, Riccardo, Giampaolo Galli, Pietro Gennari, and Fulvio Rossi, 1997, An Empirical Puzzle: Falling Migration and Growing Unemployment Differentials among Italian Regions. European Economic Review, 41 (3-5), 571-579.

[20] Friedberg, Rachel, and Jennifer Hunt, 1995, The Impact of Immigrants on Host Country Wages, Employment and Growth. Journal of Economic Perspectives 9 (2), 23-44.

[21] Funkhouser, Edward, 2000, Changes in the Geographic Concentration and Location of Residence of Immigrants. International Migration Review 34 (2), 489-510.

[22] Gregg, Paul, Stephen Machin, and Alan Manning, 2004, Mobility and Joblessness. In: David Card, Richard Blundell, and Richard Freeman, eds. Seeking a Premier Economy: The Economic Effects of British Economics Reforms 1980-2000. University of Chicago Press, 371-410.

[23] Hagen, Jürgen von, and Uwe Walz, 1994, Social Security and Migration in an Ageing Europe. CEPR Discussion Papers 1022.

[24] Hunt, Jennifer, 2006, Staunching Emigration from East Germany: Age and the Determinants of Migration. Journal of the European Economic Association 4(5) 1014-1037.

[25] Institut für Arbeitsmarkt- und Berufsforschung, 2002, IAB-Kurzbericht 25/2002, Nürnberg. 
[26] Kritz, Mary, and June Nogle, 1994, Nativity Concentration and Internal Migration among the Foreign-Born. Demography 31 (3), 509-524.

[27] LaLonde, Robert, and Robert Topel, 1997, Economic Impact of International Migration and the Economic Performance of Migrants. In M. Rosenzweig and O. Stark, eds., Handbook of Population and Family Economics, Vol. 1b , Chapter 14.

[28] Newbold, Bruce, 1999, Internal migration of the foreign-born: Population Concentration or Dispersion? Population and Environment 20 (3), 259-276.

[29] Pischke, Jörn-Steffen, and Johannes Velling, 1997, Employment Effects of Immigration to Germany: An Analysis Based on Local Labor Markets. Review of Economics and Statistics 79 (4), 594-604.

[30] Puhani, Patrick, 2001, Labour Mobility: An Adjustment Mechanism in Euroland? Empirical Evidence for Western Germany, France and Italy. German Economic Review 2 (2), 127-140.

[31] Statistisches Bundesamt, various years, Statistisches Jahrbuch für die Bundesrepublik Deutschland. (Statistical Yearbook for the Federal Republic of Germany), Wiesbaden.

[32] Treyz, George, Dan Rickman, Gary Hunt, and Michael Greenwood, 1993, The Dynamics of U.S. Internal Migration. Review of Economics and Statistics, 75 (2), 209-214. 


\section{Appendix}

\section{A State level characteristics}

\begin{tabular}{lrcccc}
\hline \hline & $\begin{array}{c}\text { population } \\
\text { (in 1000) } \\
(1998)\end{array}$ & $\begin{array}{c}\text { unemploy- } \\
\text { ment }(\%)\end{array}$ & $\begin{array}{c}\text { hourly gross } \\
\text { wage in } \\
\text { manufacturing } \\
(\text { DM), } 1998\end{array}$ & $\begin{array}{c}\text { GDP p.c. } \\
\text { (in 1000 DM) } \\
(1999)\end{array}$ & $\begin{array}{c}\text { disposable } \\
\text { income p.c. } \\
\text { (in 1000 DM) }\end{array}$ \\
\hline Baden-Württemberg & 10,476 & 8.0 & 27.94 & 54.2 & 33.3 \\
Bayern & 12,155 & 8.1 & 25.92 & 55.1 & 31.3 \\
Berlin & 3,387 & 17.9 & 26.62 & 46.8 & 27.8 \\
Brandenburg & 2,601 & 18.8 & 20.23 & 30.6 & 25.9 \\
Bremen & 663 & 16.6 & 29.69 & 64.2 & 34.0 \\
Hamburg & 1,705 & 12.7 & 29.79 & 88.8 & 32.1 \\
Hessen & 6,052 & 10.0 & 27.67 & 60.3 & 30.1 \\
Mecklenburg-Vorpommern & 1,789 & 20.5 & 19.89 & 27.8 & 24.7 \\
Niedersachsen & 7,899 & 12.3 & 29.29 & 43.2 & 29.7 \\
Nordrhein-Westfalen & 18,000 & 11.7 & 26.94 & 47.2 & 32.0 \\
Rheinland-Pfalz & 4,031 & 9.7 & 26.90 & 41.4 & 29.4 \\
Saarland & 1,072 & 12.6 & 27.57 & 44.3 & 29.3 \\
Sachsen & 4,460 & 18.8 & 19.30 & 28.8 & 25.9 \\
Sachsen-Anhalt & 2,649 & 21.7 & 19.65 & 27.5 & 25.1 \\
Schleswig-Holstein & 2,777 & 11.2 & 26.32 & 43.9 & 30.1 \\
Thüringen & 2,449 & 18.3 & 18.65 & 27.9 & 25.7 \\
\hline
\end{tabular}

Source: Arbeitskreis VGR (2002) and Institut der Deutschen Wirtschaft (1999, 2000)

Table 14: German labor market statistics, by federal state 


\begin{tabular}{lr}
\hline \hline $\begin{array}{l}\text { largest groups of resident non-nationals (1998) } \\
\text { nationality }\end{array}$ & \\
total number (in 1000) & $2,110.2$ \\
Yurkey & 719.5 \\
Italy & 612.0 \\
Greece & 363.5 \\
Poland & 283.6 \\
Croatia & 208.9 \\
Bosnia and Herzegovina & 190.1 \\
Austria & 185.2 \\
Portugal & 132.6 \\
Spain & 131.1 \\
Russian Federation & 125.1 \\
Iran & 115.1 \\
UK & 114.1 \\
Netherlands & 112.1 \\
United States & 110.7 \\
France & 105.8 \\
\hline Source: Statistisches Bundesamt (2000, table 3.21, p. 65$)$
\end{tabular}

Table 15: The largest groups by nationality

\begin{tabular}{lrc}
\hline \hline & $\begin{array}{c}\text { total foreign population } \\
\text { (in 1000, 12/1998) }\end{array}$ & $\begin{array}{c}\text { percentage of foreign population } \\
\text { in total population }(6 / 1999)\end{array}$ \\
\hline Baden-Württemberg & 1,267 & 12.5 \\
Bayern & 1,096 & 9.3 \\
Berlin & 482 & 12.8 \\
Brandenburg & 54 & 2.3 \\
Bremen & 82 & 15.2 \\
Hamburg & 319 & 15.2 \\
Hessen & 842 & 12.2 \\
Mecklenburg-Vorpommern & 26 & 1.7 \\
Niedersachsen & 474 & 6.7 \\
Nordrhein-Westfalen & 1,995 & 11.4 \\
Rheinland-Pfalz & 299 & 7.7 \\
Saarland & 80 & 8.1 \\
Sachsen & 83 & 2.3 \\
Sachsen-Anhalt & 47 & 1.6 \\
Schleswig-Holstein & 143 & 5.4 \\
Thüringen & 31 & 1.6 \\
\hline Germany total & 7,320 & 9.0 \\
\hline
\end{tabular}

Source: Statistisches Jahrbuch (1999)

Table 16: Foreigners by federal state 


\section{B Descriptive evidence using official registration records}

In this appendix I describe differences between natives and foreigners with respect to migration across Gemeinde- (town) borders, Kreis- (county) borders and Länder- (federal state) borders. ${ }^{32}$ There are 16 Länder, 439 Kreise and several thousand Gemeinden. For the tables referring to migration across Kreis- and Gemeinde-borders below I calculate for each state the share of natives and foreigners that move relative to the total number of natives and foreigners. The data refer to the year 2000 and are from the Statistisches Bundesamt (German Federal Statistical Office). As explained above the data cover all individuals living in Germany, but have the disadvantage that they record foreigners as those who do not have German citizenship, and therefore cannot distinguish between foreigners born in Germany and foreigners that immigrated from abroad.

The results (table 17) indicate that independent of the specific regional category considered, the share of foreigners who migrate within Germany is larger than the share of natives who migrates internally: within the group of natives $4.6 \%$ move across Gemeinde-borders, while $6.5 \%$ of foreigners migrate over Gemeinde-borders. If migration across Kreis-borders is considered, i.e. migration to a place further away from the origin, naturally the migration rates are reduced, with the numbers being $3.1 \%$ for natives and $5.0 \%$ for foreigners. Looking across states, it is clear that the result at the federal level does not depend on particular states, but that foreigners are more mobile than natives in 14 of the 16 German states. Finally, consider migration across the border of the state (Bundesland). This type of migration spans the longest distances. Again, foreigners are more likely to migrate than natives in Germany, with $1.9 \%$ of all foreigners but only $1.3 \%$ of natives migrating. Again, the result at the federal level does not depend on particular states: foreigners are more mobile than natives in 13 of the 16 German states.

\footnotetext{
${ }^{32}$ More specifically, in this section I study out-migration, i.e. migration away from the place of origin, as opposed to in-migration, i.e. migration to the destination.
} 


\begin{tabular}{lcccccc}
\hline \hline & \multicolumn{5}{c}{ \% that move over borders of: } \\
& \multicolumn{1}{c}{ Gemeinde (town) } & Kreis & (county) & \multicolumn{2}{c}{ Bundesland (state) } \\
& Natives & Foreigner & Natives & Foreigner & Natives & Foreigner \\
\hline Baden-Württemberg & 5.02 & 7.28 & 3.04 & 4.66 & 1.0 & 1.3 \\
Bayern & 4.52 & 7.14 & 3.11 & 5.64 & 0.7 & 1.3 \\
Berlin & 2.54 & 1.73 & 2.54 & 1.73 & 2.5 & 1.7 \\
Brandenburg & 5.47 & 14.50 & 3.45 & 11.70 & 2.1 & 6.5 \\
Bremen & 3.47 & 3.47 & 3.47 & 3.47 & 3.4 & 3.3 \\
Hamburg & 3.21 & 1.96 & 3.21 & 1.96 & 3.2 & 2.0 \\
Hessen & 4.70 & 7.47 & 3.06 & 4.92 & 1.3 & 1.8 \\
Mecklenburg-Vorpommern & 5.76 & 20.39 & 3.95 & 17.45 & 1.9 & 7.6 \\
Niedersachsen & 5.77 & 9.77 & 4.06 & 7.84 & 2.2 & 4.0 \\
Nordrhein-Westfalen & 3.71 & 4.73 & 2.78 & 3.93 & 0.8 & 0.9 \\
Rheinland-Pfalz & 5.45 & 11.17 & 3.45 & 8.68 & 1.4 & 3.0 \\
Saarland & 3.99 & 6.71 & 2.30 & 4.71 & 1.0 & 2.2 \\
Sachsen & 4.04 & 10.56 & 2.82 & 9.31 & 1.4 & 5.7 \\
Sachsen-Anhalt & 4.85 & 18.21 & 3.10 & 13.98 & 1.8 & 10.9 \\
Schleswig-Holstein & 6.35 & 8.83 & 3.72 & 6.46 & 1.7 & 3.0 \\
Thüringen & 3.92 & 16.71 & 2.60 & 14.52 & 1.5 & 6.3 \\
\hline Germany & 4.56 & 6.55 & 3.12 & 4.96 & 1.3 & 1.9 \\
\hline source: Statistisches Bundesamt & & & & & \\
\hline
\end{tabular}

Table 17: Internal migration (\% of natives and foreigners that move over town/county/state borders, data is for 2000) 


\section{Further robustness checks with the "household head/labor market participants" sample}

\begin{tabular}{|c|c|c|c|}
\hline \multicolumn{4}{|c|}{ Dependent variable $=1$ if individual moved to a different federal state over the previous year } \\
\hline & (1) & (2) & (3) \\
\hline & all & all & all \\
\hline immigrant & $\begin{array}{l}0.002 \\
(0.001)^{* *}\end{array}$ & $\begin{array}{l}0.002 \\
(0.001)^{* *}\end{array}$ & \\
\hline $\begin{array}{l}\text { recent immigrant } \\
\quad(<10 \mathrm{yrs})\end{array}$ & & & $\begin{array}{l}0.004 \\
(0.001)^{* *}\end{array}$ \\
\hline age $(/ 100)$ & $\begin{array}{l}-0.191 \\
(0.009)^{* *}\end{array}$ & $\begin{array}{l}-0.115 \\
(0.009)^{* *}\end{array}$ & $\begin{array}{l}-0.114 \\
(0.009)^{* *}\end{array}$ \\
\hline age squared & $\begin{array}{l}0.155 \\
(0.011)^{* *}\end{array}$ & $\begin{array}{l}0.067 \\
(0.011)^{* *}\end{array}$ & $\begin{array}{l}0.067 \\
(0.011)^{* *}\end{array}$ \\
\hline married & $\begin{array}{l}-0.004 \\
(0.000)^{* *}\end{array}$ & $\begin{array}{l}0.002 \\
(0.000)^{* *}\end{array}$ & $\begin{array}{l}0.002 \\
(0.000) * *\end{array}$ \\
\hline widowed & $\begin{array}{l}-0.002 \\
(0.001)^{* *}\end{array}$ & $\begin{array}{l}0.000 \\
(0.001)\end{array}$ & $\begin{array}{l}0.000 \\
(0.001)\end{array}$ \\
\hline divorced & $\begin{array}{l}0.001 \\
(0.000)\end{array}$ & $\begin{array}{l}0.002 \\
(0.000)^{* *}\end{array}$ & $\begin{array}{l}0.002 \\
(0.000) * *\end{array}$ \\
\hline finished school & $\begin{array}{l}0.003 \\
(0.002)\end{array}$ & $\begin{array}{l}0.003 \\
(0.002)\end{array}$ & $\begin{array}{l}0.003 \\
(0.002)\end{array}$ \\
\hline vocational training & $\begin{array}{l}0.001 \\
(0.001)\end{array}$ & $\begin{array}{l}0.002 \\
(0.001)\end{array}$ & $\begin{array}{l}0.002 \\
(0.001)\end{array}$ \\
\hline higher voc. training & $\begin{array}{l}0.003 \\
(0.002)\end{array}$ & $\begin{array}{l}0.003 \\
(0.002)^{*}\end{array}$ & $\begin{array}{l}0.003 \\
(0.002)^{*}\end{array}$ \\
\hline tertiary degree & $\begin{array}{l}0.015 \\
(0.003)^{* *}\end{array}$ & $\begin{array}{l}0.012 \\
(0.002) * *\end{array}$ & $\begin{array}{l}0.011 \\
(0.002) * *\end{array}$ \\
\hline unempl. last yr & & $\begin{array}{l}0.006 \\
(0.001)^{* *}\end{array}$ & $\begin{array}{l}0.006 \\
(0.001)^{* *}\end{array}$ \\
\hline $\begin{array}{l}\text { household size } \\
\text { indicators (10) }\end{array}$ & & yes & yes \\
\hline $\begin{array}{l}\text { income category } \\
\text { indicators }(10)\end{array}$ & & yes & yes \\
\hline observations & 547209 & 532346 & 532346 \\
\hline log-likelihood & -32939.6 & -29181.1 & -29177.6 \\
\hline
\end{tabular}

Table 18: Reduced form results from the microcensus: moves across federal states, robustness checks with the "household head/labor market participants" sample (for sample restrictions details see text) 